2

17 *corresponding author

\title{
Enhanced susceptibility of methylmercury bioaccumulation into seston of the Laurentian Great Lakes
}

\section{${ }^{b}$ U.S. Environmental Protection Agency Office of Research and Development, Center for Computational Toxicology and Exposure, Great Lakes Toxicology and Ecology Division, 6201 Congdon Blvd, Duluth, MN 55804, USA}

cUniversity of Wisconsin Aquatic Sciences Center, 1975 Willow Dr., Madison, WI 53706

dEnvironmental Chemistry and Technology program, University of Wisconsin-Madison, Madison, WI 53706

19 Any use of trade, firm, or product names in this publication is for descriptive purposes only and 20 does not imply endorsement by the U.S. Government. 
21 S2 to S3 - Text.

$22 \mathrm{~S} 4$ to S8 - Figures S1 - S5 - Sample site locations.

23 S9 - Figure S6 - Lake Erie nearshore, western, central, and eastern UTHg concentrations.

24 S10 - Figure S7 - Lake Erie nearshore, western, central, and eastern DOC and FMeHg

25 concentrations.

26 S11 - Figure S8 - Percent of UMeHg as PMeHg versus PMeHg.

27 S12 - Figure S9 - Comparison of fall time nearshore and offshore seston from intense cruises.

28 S13 - Figure S10 - Seston carbon to nitrogen ratios.

29 S14 - Figure S11 - Seston carbon content relative to carbon to nitrogen mass ratios.

$30 \mathrm{~S} 15$ - Figure S12 - Seston carbon content relative to BMeHg.

31 S16 - Figure S13 - Nitrogen stable isotope ratio relative to carbon normalized seston BMeHg.

32 S17 - Figure S14 - Nitrogen stable isotope ratio relative to lipid-corrected carbon stable isotope 33 ratio.

34 S18 - Figure S15 - Carbon and nitrogen isotope ratios, $\mathrm{C}: \mathrm{N}$, and BMeHg relative to dry-weight 35 biomass.

36 S19 - Figure S16 - Physical and mercury concentration characterization of surficial sediments.

37 S20 - Figure S17 - Mercury concentrations in composited mussel tissue.

38 S20 - Figure S18 - SUVA $254 \mathrm{~nm}$ values for waters collected during the 2011 spring cruises.

39 S21 to S31 - Table S1 - Methodological details for analytical constituents.

40 S32 to S36 - Table S2 - S6 - Site coordinates for Lakes Erie, Huron, Michigan, Ontario and 41 Superior.

42 S37 to S39 - Table S7 - Sediment total mercury, methylmercury, and loss on ignition.

43 S40 - Table S8 - PHgT concentrations, PMeHg concentrations, $\log \mathrm{K}_{\mathrm{d}}$, and aqueous DOC

44 concentrations.

45 S41 - Table S9 - Annual summary of methods employed, analytes measured, and data used.

46 S42 - Table S10 - Interlaboratory comparison of carbon and nitrogen isotopes in seston material.

47 S42 to S43 - Citations

48 
50 Surficial sediments were collected onboard the $R / V$ Lake Guardian during the summer and fall

51 surveys $(2010$ - 2014). A Ponar dredge was used to retrieve the sediment from the lake bottom.

52 Once onboard, the top $2 \mathrm{~cm}$ of intact sediment was subsampled into an acid-cleaned Teflon jar

53 and immediately frozen. At the U.S. Geological Survey Mercury Research Laboratory (USGS

54 MRL), the sediments were lyophilized and homogenized by ball milling. Total mercury and

55 methylmercury concentrations were measured in sediments using adaptations of U.S.

56 Environmental Protection Agency (US EPA) methods 7473 and 1630, respectively., 2 The mass-

57 percent of dry mass in sediment was measured by comparing the wet mass and dry mass of

58 sediment following lyophilization. The mass-percent of combustible carbon $\left(550{ }^{\circ} \mathrm{C}\right.$ for $\left.2 \mathrm{~h}\right)$ was

59 measured in lyophilized sediment and reported as percent sediment carbon lost on ignition.

60 Quality assurance and control measurements for sediment mercury analyses were within the

61 guidelines of the USGS MRL quality assurance plan (summarized in Table S1), or otherwise the

62 data were not reported or appropriately flagged in the data release

63 (https://doi.org/10.5066/P9GEIVXD). ${ }^{3}$

64 Mussels

65 When present, mussels (genus Dreissena) were collected from sediment Ponar dredges in

66 summer and fall cruises $(2010$ - 2013) in all lakes except Lake Superior. Following collection,

67 the mussels were rinsed of excess sediment using surficial lake water and then frozen intact in

68 Teflon sampling jars. For the summer survey in 2010 and Lake Michigan fall sampling in 2013,

69 mussels were first size separated by maximum valve length and then binned into $2.6 \mathrm{~mm}$ and 7

$70 \mathrm{~mm}$ (respectively) size cohorts and composited as individual samples. Because the size separation

71 of mussels did not indicate that mercury concentrations were length-dependent (Figure S16),

72 mussels collected in the remaining summer surveys (2011 - 2013) were not size separated;

73 however, only organisms ranging 10-30 $\mathrm{mm}$ were retained. Prior to analysis, the mussel tissue 
74 was removed from the shell, lyophilized, and homogenized by ball milling. Quality assurance and

75 control measurements for biological mercury analyses were within the guidelines of the USGS

76 MRL quality assurance plan (summarized in Table S1), or otherwise the data were not reported or

77 appropriately flagged in the data release (https://doi.org/10.5066/P9GEIVXD). ${ }^{3}$

\section{$78 \quad$ Statistical approaches}

79 All statistical analyses were performed using Sigmaplot 14.0, and two-dimensional graphs were

80 designed there as well. Maps were created using ESRI ArcMap 10.8.1 on Windows 7. All raw

81 data can be found in the associated data release (https://doi.org/10.5066/P9GEIVXD). ${ }^{3}$ Use of

82 that data may cite this primary manuscript. 
83 Figure S1. Sample site locations for Lake Erie.

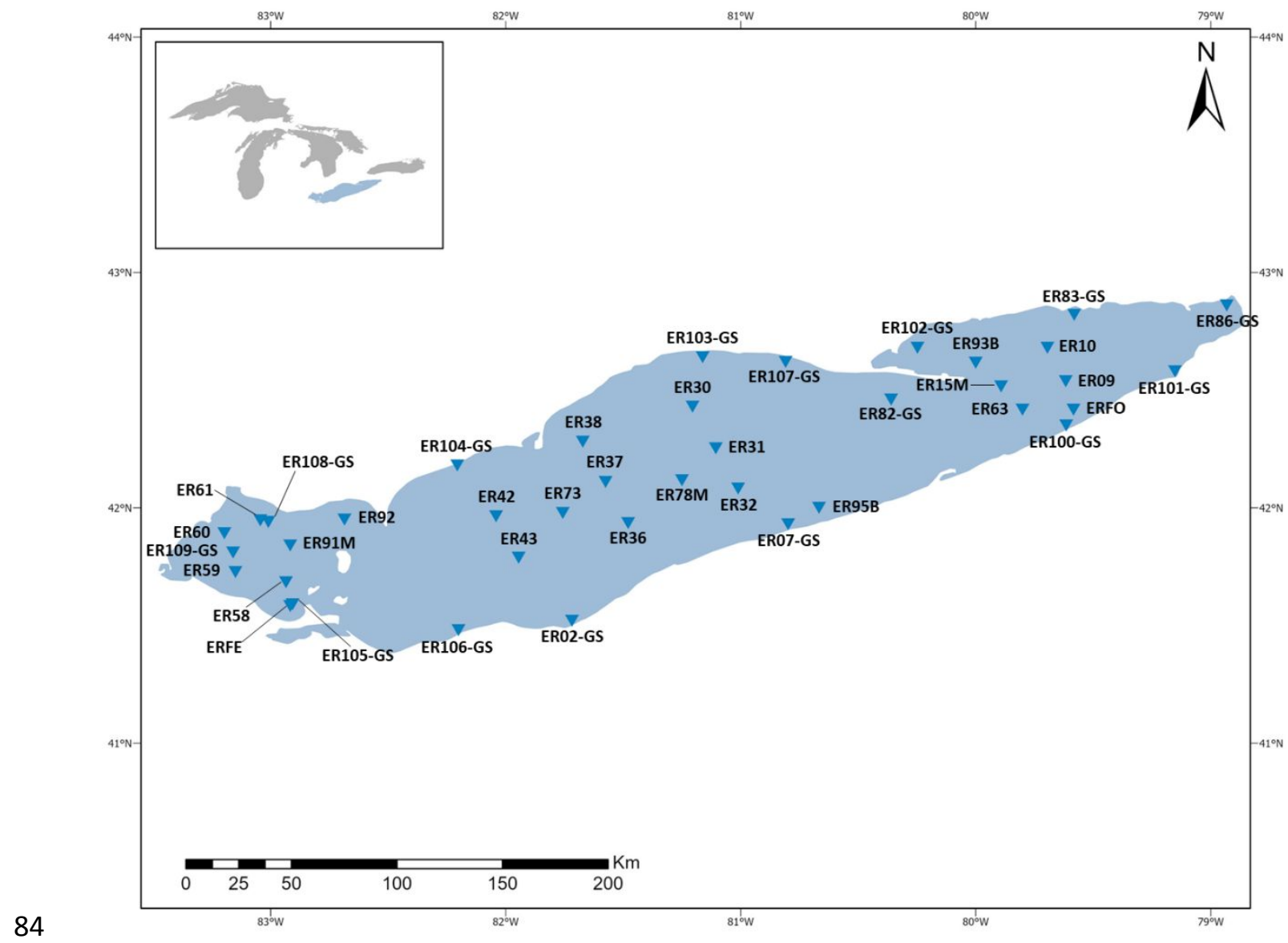

85

86 
87 Figure S2. Sample site locations for Lake Huron.

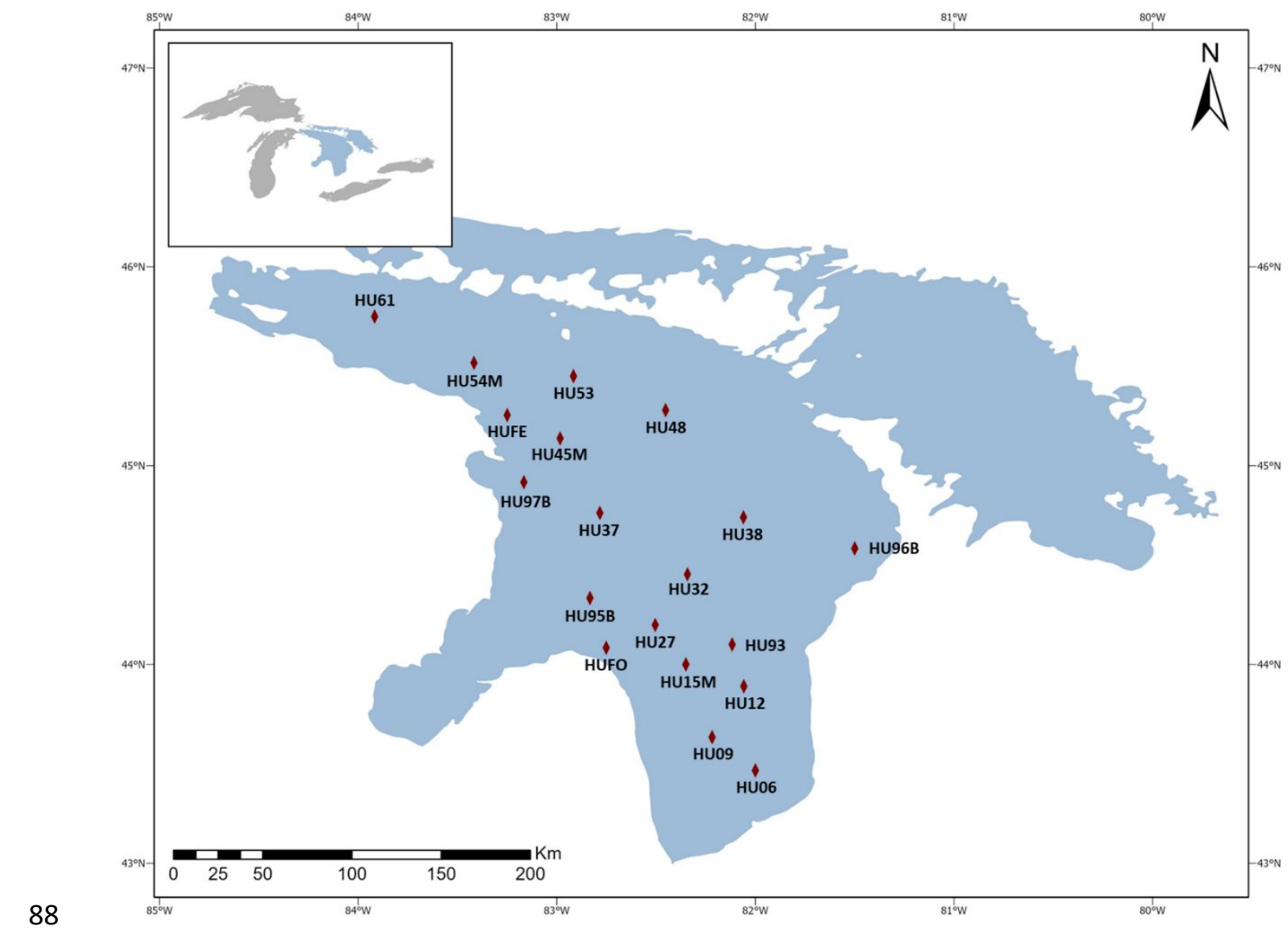

89 
Figure S3. Sample site locations for Lake Michigan.

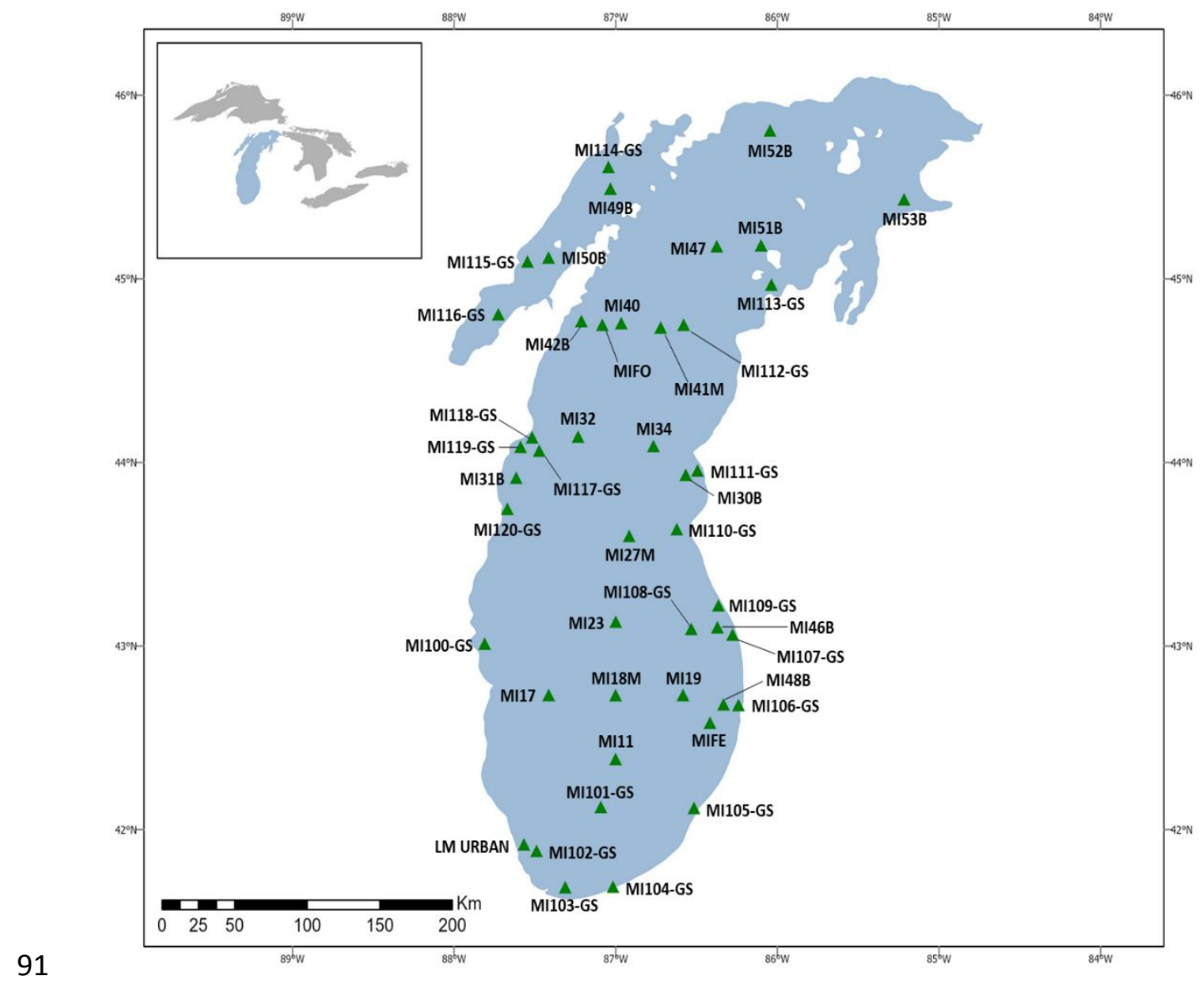

92 
93 Figure S4. Sample site locations for Lake Ontario.

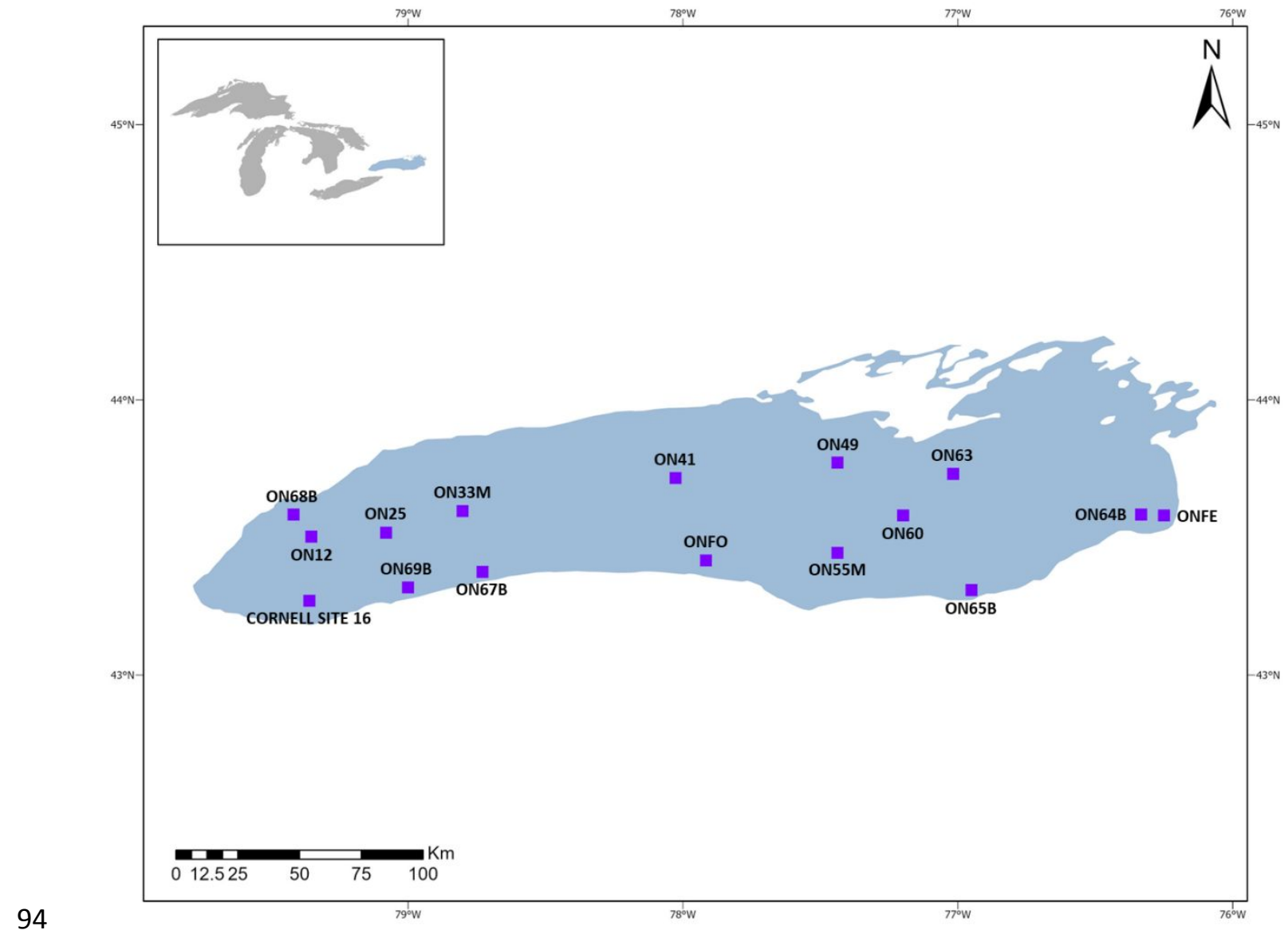


96 Figure S5. Sample site locations for Lake Superior.

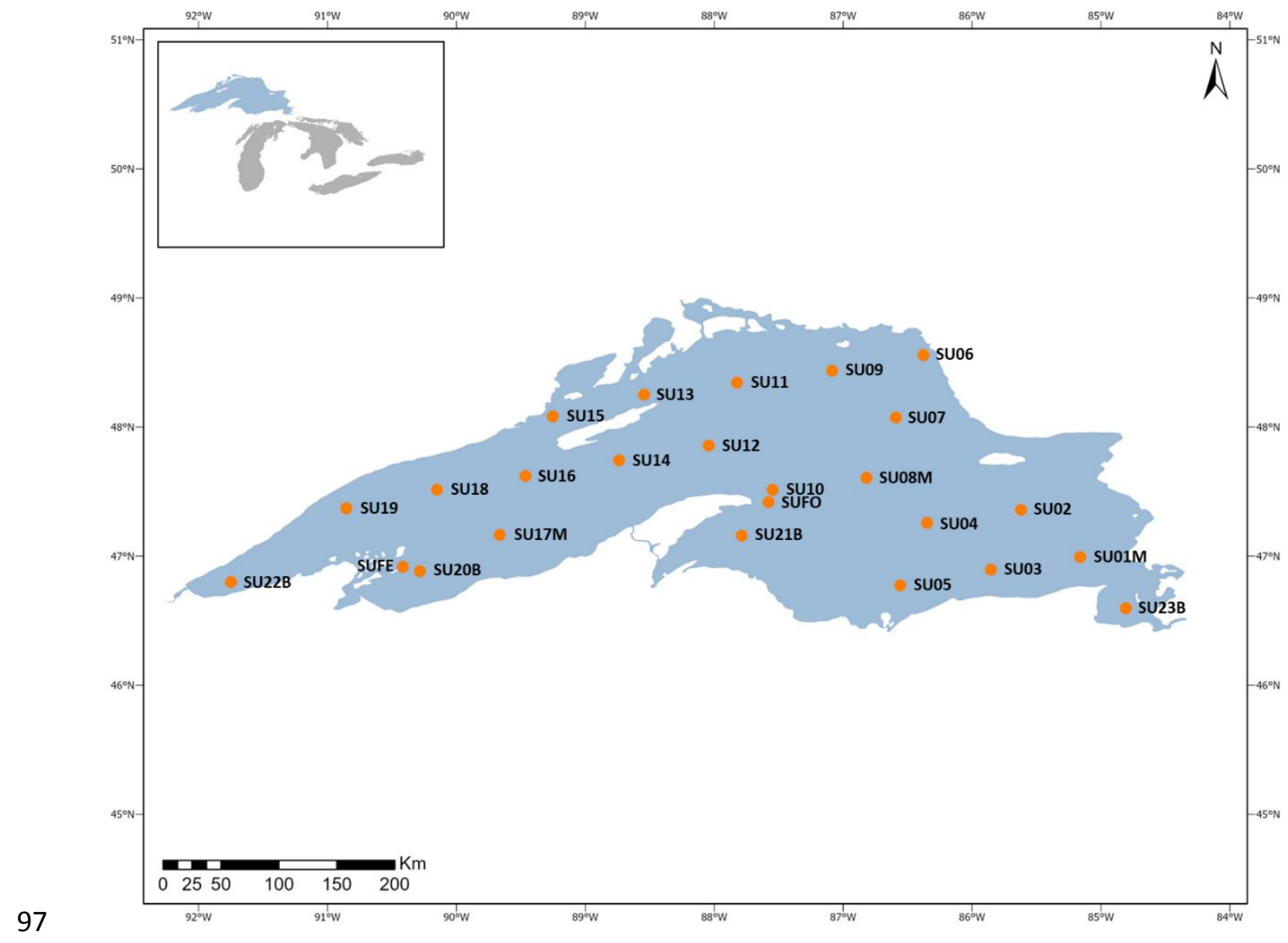

98 
Figure S6. Aqueous concentrations of unfiltered total mercury (UHgT) as a raw-water measurement or as the summed value of filter-passing and particulate-bound mercury $(\mathrm{FT}+\mathrm{PT})$ at operationally defined depth layers for the nearshore (NS), eastern, central, and western Lake Erie basins. Inner box lines represent data median, the box edges represent 25 th and 75 th percentiles, the edge of the whiskers represent the 10th and 90th percentiles, and the triangles represent outliers. Sampling season (spring, summer, or fall) and year (2010-2014, and 2018) are described 106 on the $\mathrm{x}$-axis.

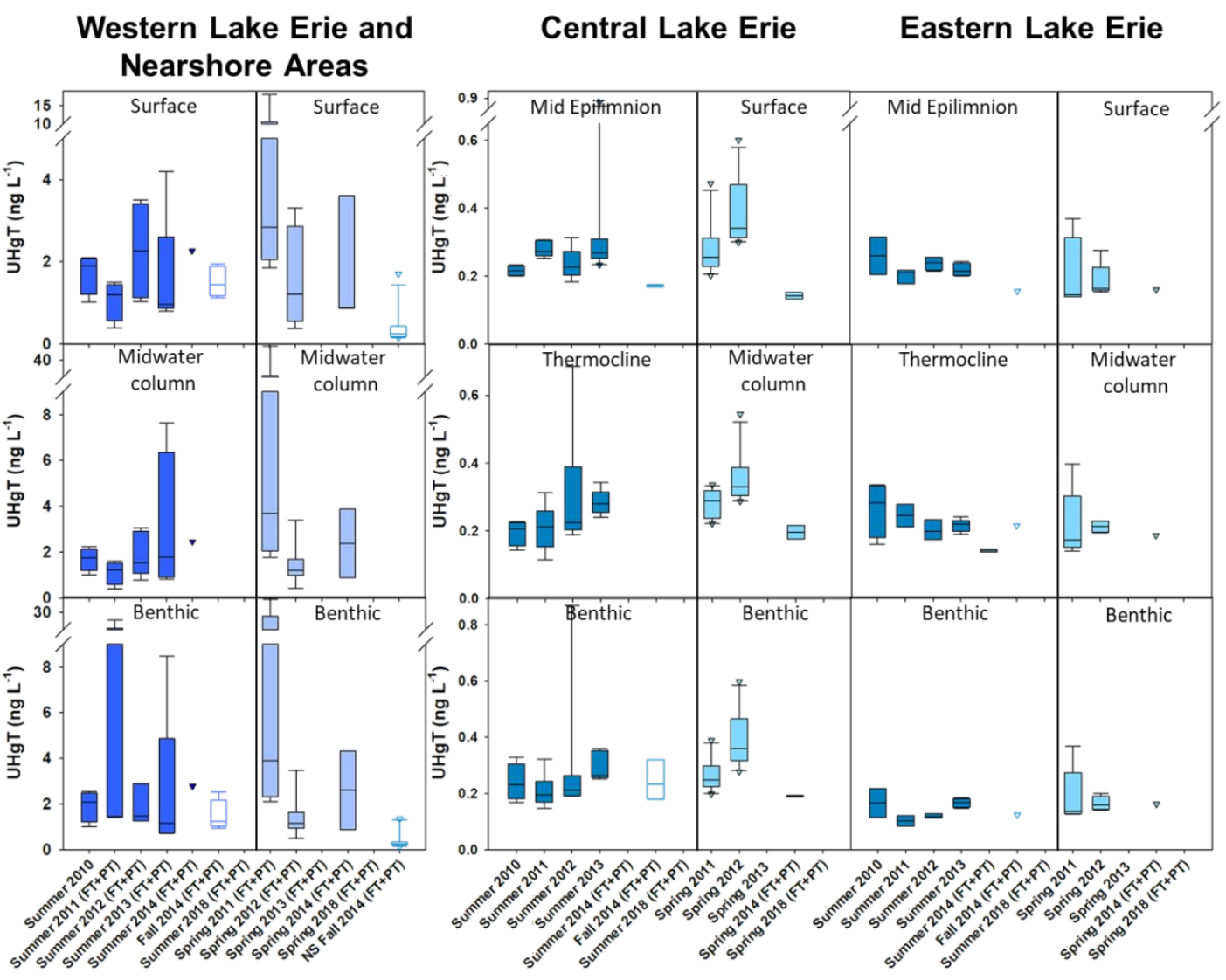


Figure S7. Aqueous concentrations of dissolved organic carbon (DOC-blue boxplots) and filterpassing methylmercury (FMeHg-gray boxplots) at operationally defined depth layers for the nearshore (NS), eastern, central, and western Lake Erie basins. Inner box lines represent data median, the box edges represent 25 th and 75 th percentiles, the edge of the whiskers represent the 10 th and 90th percentiles, and the triangles represent outliers. Sampling season (spring, summer, or fall) and year (2010-2014, and 2018) are described on the $\mathrm{x}$-axis.

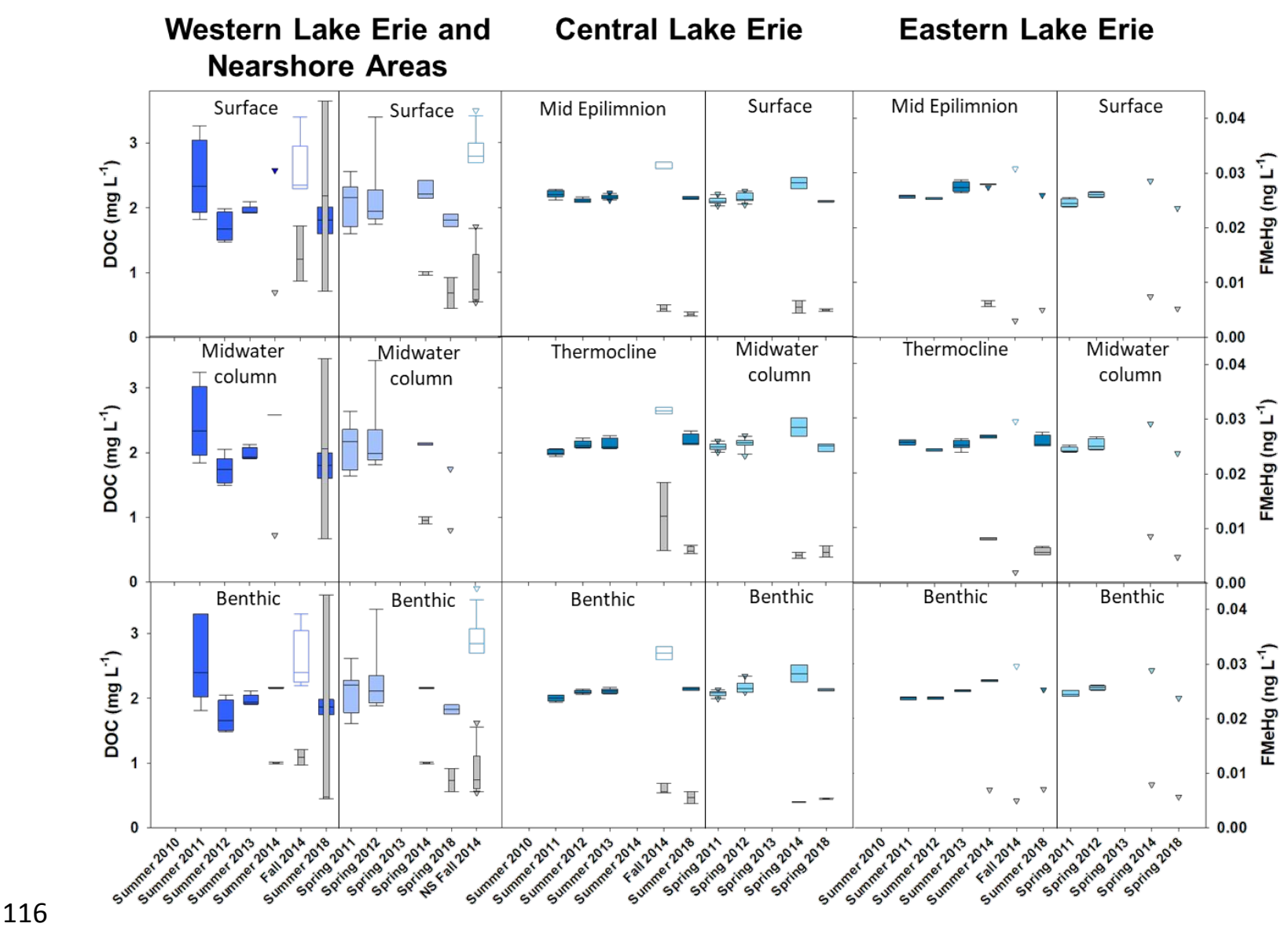


133 Figure S8. Average percent of unfiltered methylmercury (UMeHg) as particulate-bound 134 methylmercury (PMeHg) relative to $\mathrm{PMeHg}$ concentration in water. Summer samples are 135 represented by a darker shade datapoint relative to samples collected in spring. Error bars 136 represent one standard deviation.

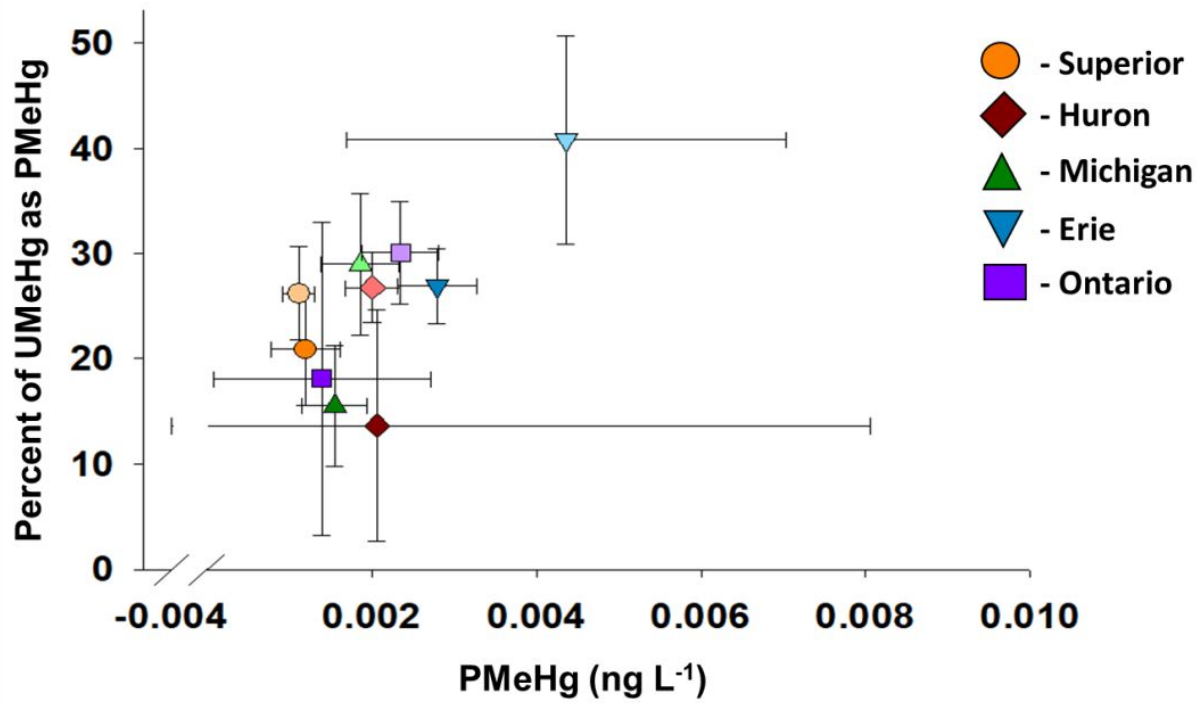


167 Figure S9. Paired nearshore and offshore size-sieved seston for the fall-time sampling of Lake 168 Michigan (2013) and Lake Erie (2014). Inner box lines represent data median, the box edges represent 25th and 75th percentiles, the edge of the whiskers represent the 10th and 90th percentiles, and the triangles represent outliers. Sizes are denoted and "Yes" indicates by MannWhitney Rank Sum Test or Student T-Test a significant difference $(\mathrm{p}<0.05)$ between offshore and nearshore seston.

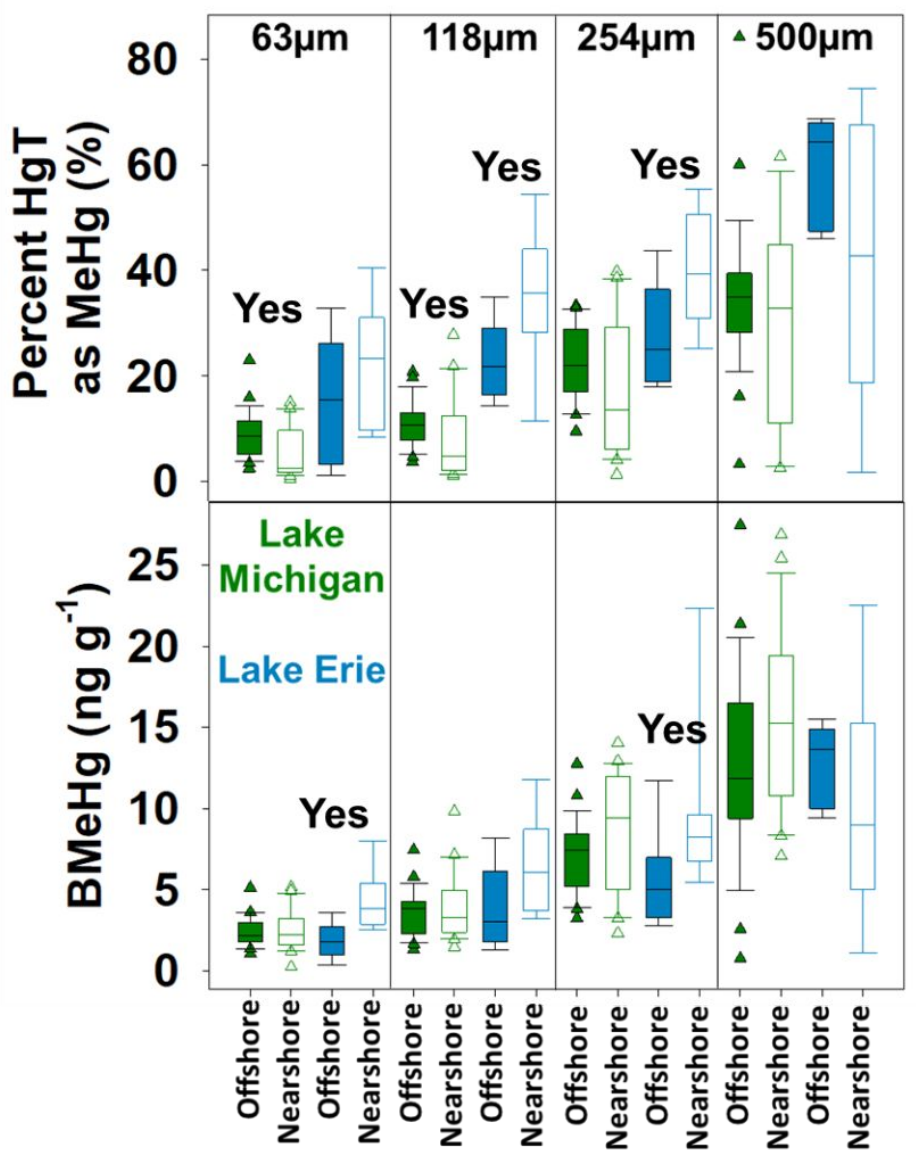


174 Figure S10. Carbon to nitrogen ratios in seston by lake and size fraction $(63=63-118 \mu \mathrm{m}, 118=$ $175118-243 \mu \mathrm{m}, 243=243-500 \mu \mathrm{m}$, and $500=>500 \mu \mathrm{m})$. Inner box lines represent data median, the 176 box edges represent 25th and 75th percentiles, the edge of the whiskers represent the 10th and 177 90th percentiles, and the triangles represent outliers.

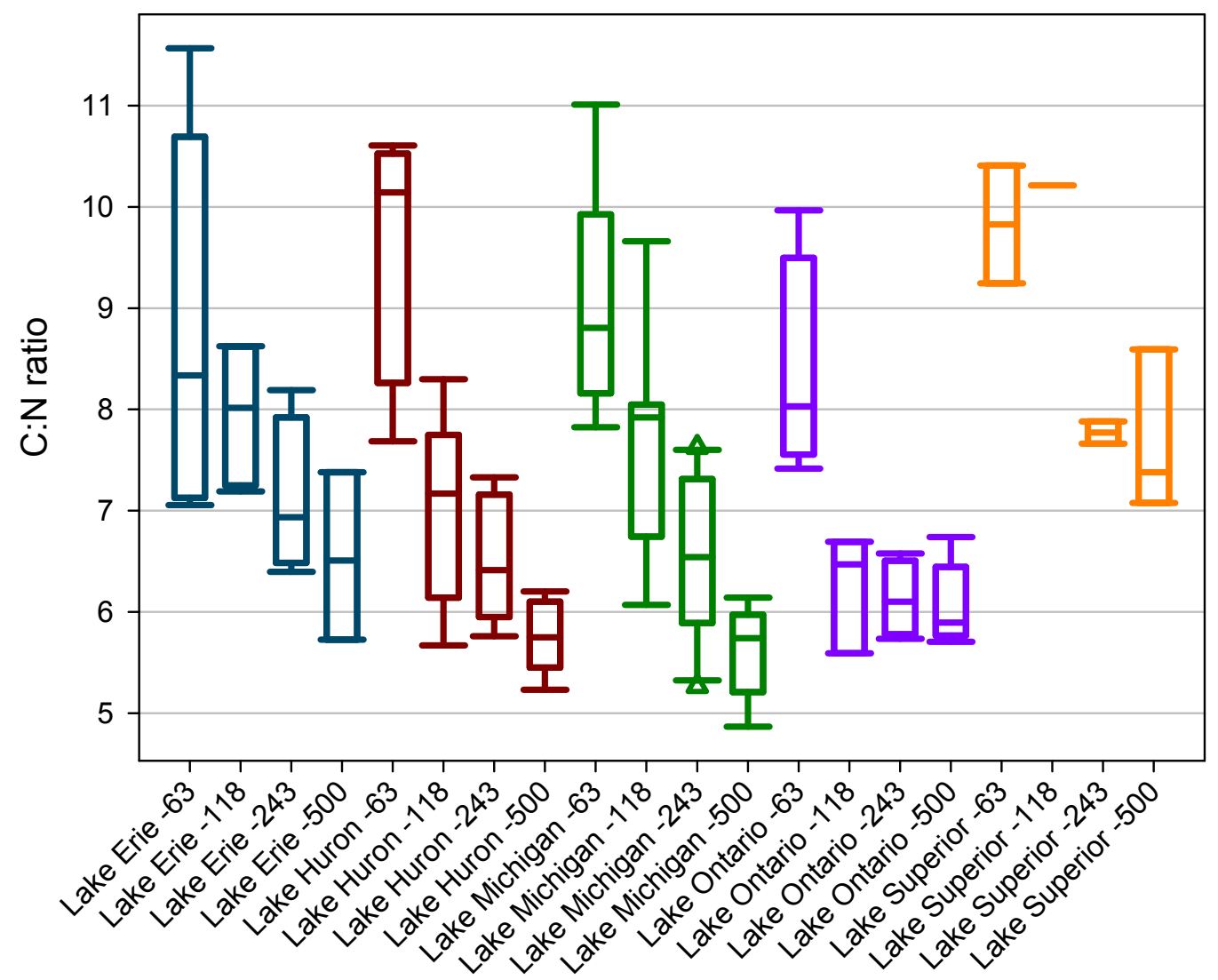


180 Figure S11. Percent of seston tissue as carbon relative to carbon to nitrogen mass ratio in size181 sieved seston samples. Summer samples are represented by a darker shade datapoint relative to 182 samples collected in spring. Relative datapoint size indicates seston size fraction $(63-118 \mu \mathrm{m}$, $183 \quad 118-243 \mu \mathrm{m}, 243-500 \mu \mathrm{m},>500 \mu \mathrm{m})$.

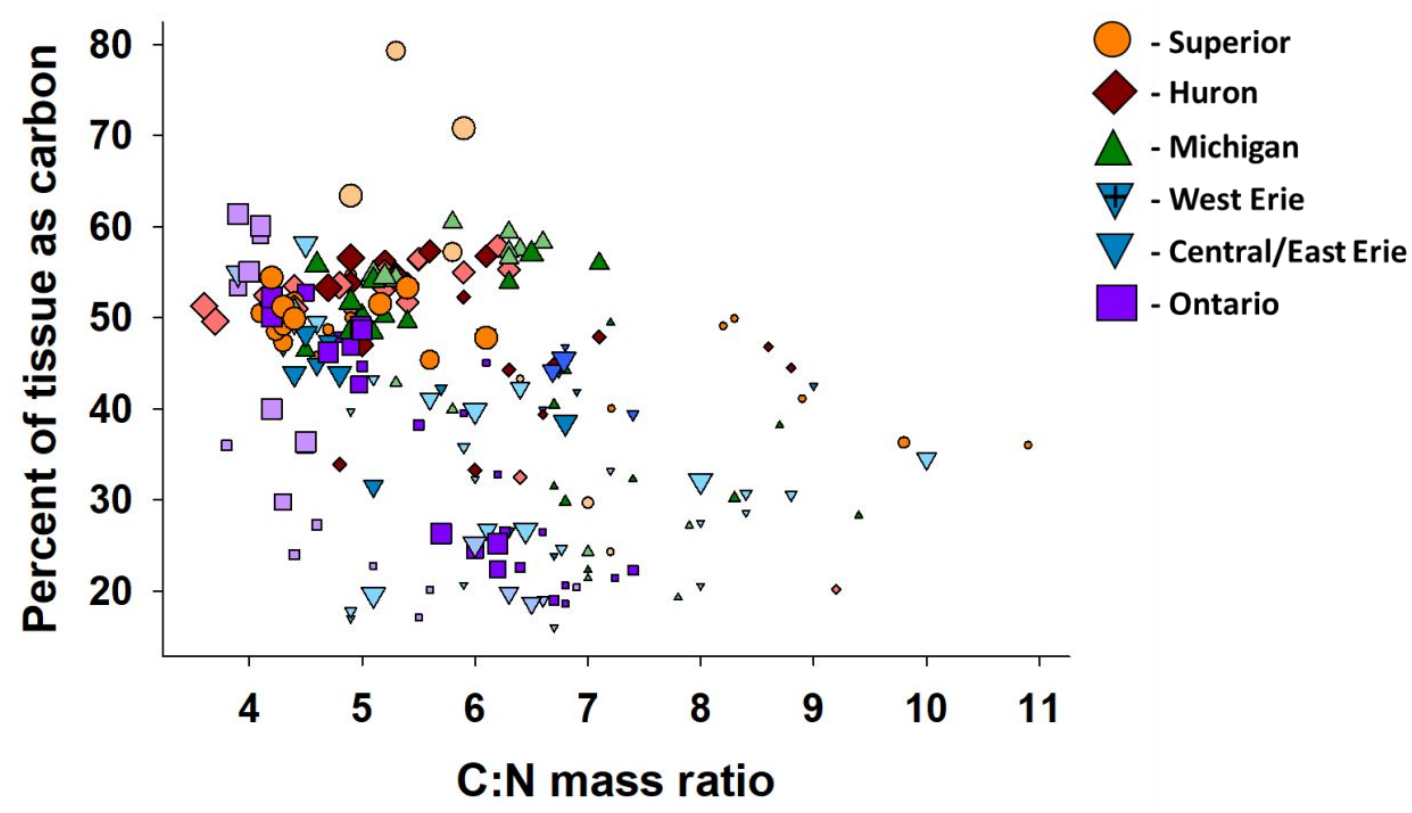


185 Figure S12. Percent of seston tissue as carbon relative to methylmercury concentration (BMeHg).

186 Summer samples are represented by a darker shade datapoint relative to samples collected in

187 spring. Relative datapoint size indicates seston size fraction $(63-118 \mu \mathrm{m}, 118-243 \mu \mathrm{m}, 243-500$

$188 \mu \mathrm{m},>500 \mu \mathrm{m})$.

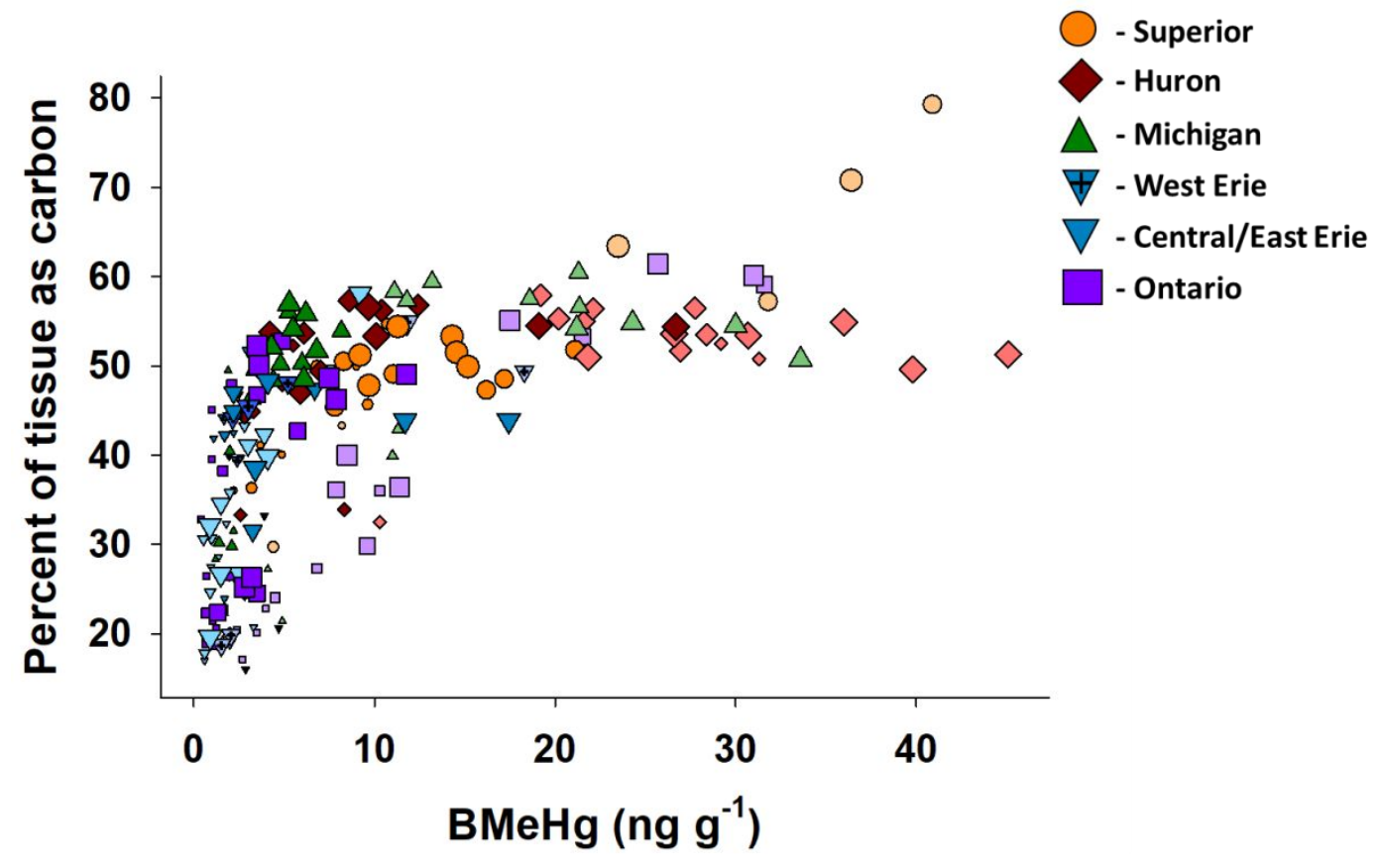

190 
Figure S13. (A) Nitrogen stable isotope ratio $\left(\delta^{15} \mathrm{~N}\right)$ relative to carbon normalized seston methylmercury concentration (BMHG) as dry weight (DW) in seston tissue. (B) $\delta^{15} \mathrm{~N}$ of larger seston minus $\delta^{15} \mathrm{~N}$ of $63-118 \mu \mathrm{m}$ seston relative to carbon normalized seston BMeHg as DW. Spring samples are represented by a lighter shade datapoint relative to samples collected in summer. Relative datapoint size indicates seston size fraction (63-118 $\mu \mathrm{m}, 118-243 \mu \mathrm{m}, 243-500 \mu \mathrm{m},>500 \mu \mathrm{m}$ ). (C) Linear regression (solid line) and 95\% confidence interval for the paired $\delta^{15} \mathrm{~N}$ (normalized to $63-118 \mu \mathrm{m}$ seston) and carbon normalized seston BMeHg biomagnification factor (BMF, normalized to $63-118 \mu \mathrm{m}$ seston) by lake. Except for Lake Ontario spring, seasons were not distinguishable and so they were merged. No linear regression is statistically dissimilar from others except for Lake Ontario spring.
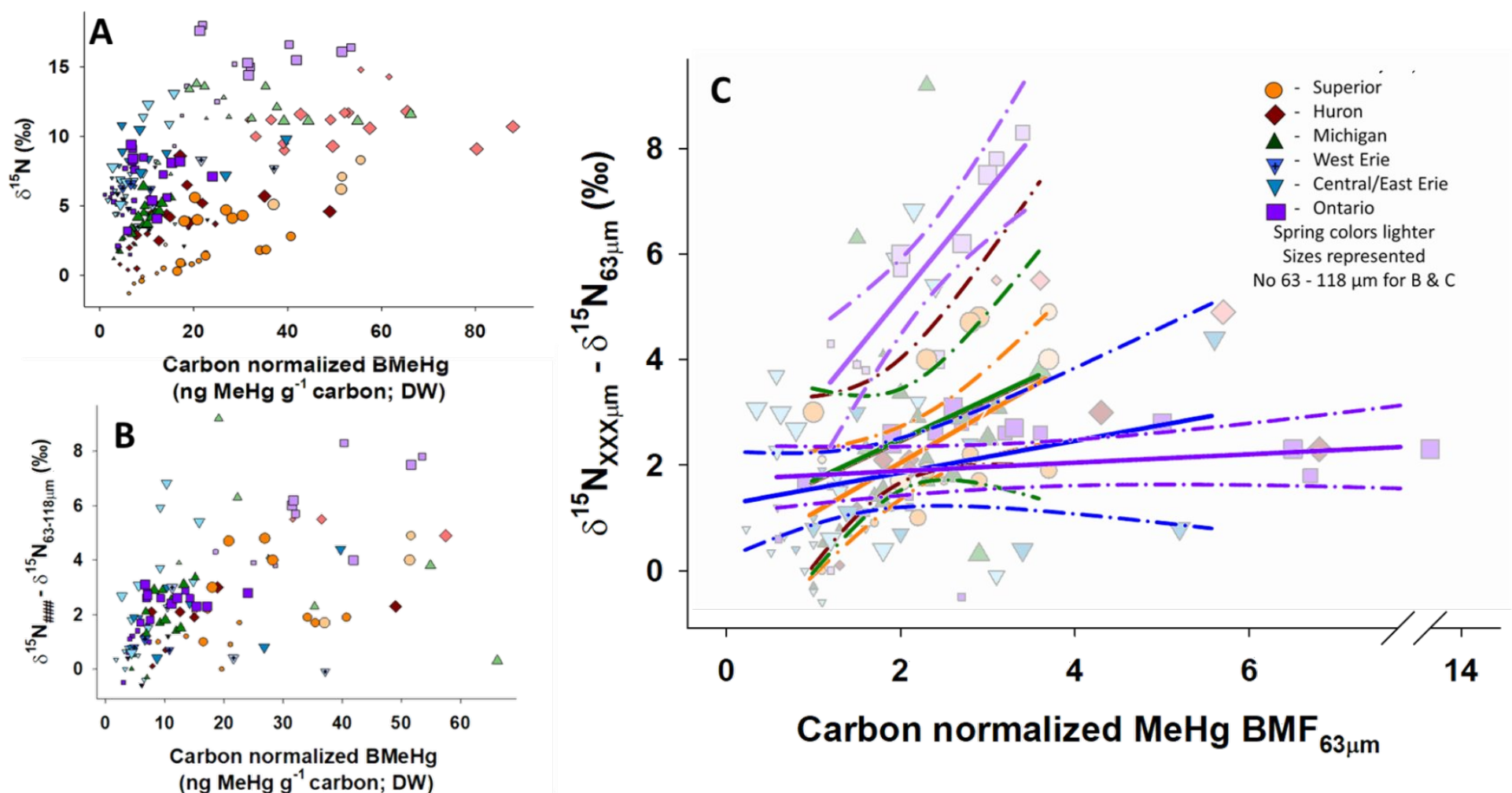
199 Figure S14. Nitrogen stable isotope ratio $\left(\delta^{15} \mathrm{~N}\right)$ relative to lipid-corrected carbon stable isotope 200 ratio $\left(\delta^{13} \mathrm{C}\right)$ in the 63-118 $\mu \mathrm{m}(\mathrm{A}), 118-243 \mu \mathrm{m}(\mathrm{B}), 243-500 \mu \mathrm{m}(\mathrm{C})$, and $>500 \mu \mathrm{m}$ (D) seston 201 size fractions. Spring samples are represented by a lighter shade datapoint relative to samples 202 collected in summer.
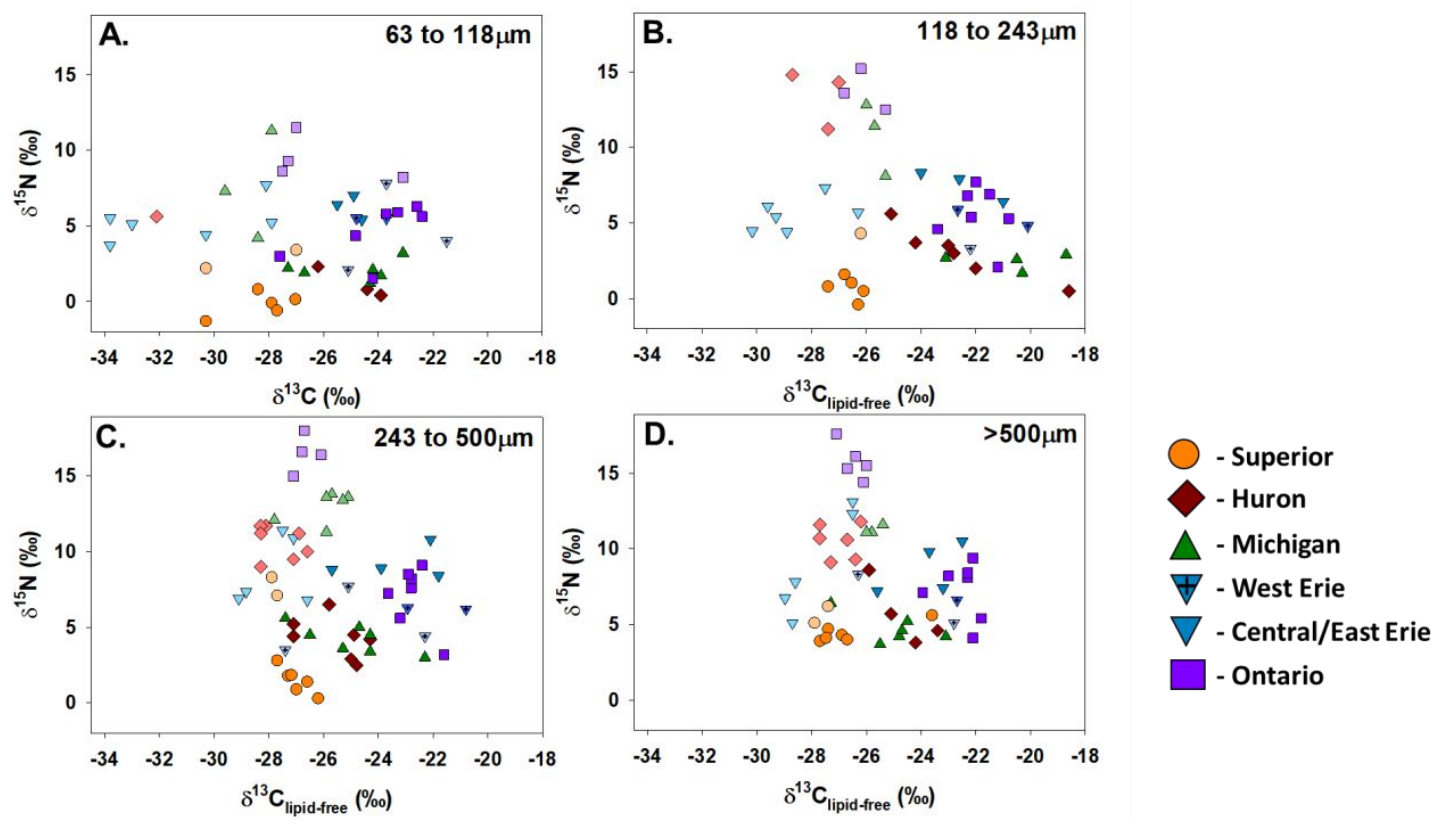
Figure S15. Nitrogen stable isotope ratio $\left(\delta^{15} \mathrm{~N}\right)$ in seston tissue relative to dry-weight biomass in the 63-118 $\mu \mathrm{m}$ (A), 118-243 $\mu \mathrm{m}$ (B), 243-500 $\mu \mathrm{m}$ (C), and $>500 \mu \mathrm{m}$ (D) seston size fractions. Spring samples are represented by a lighter shade datapoint relative to samples collected in summer.
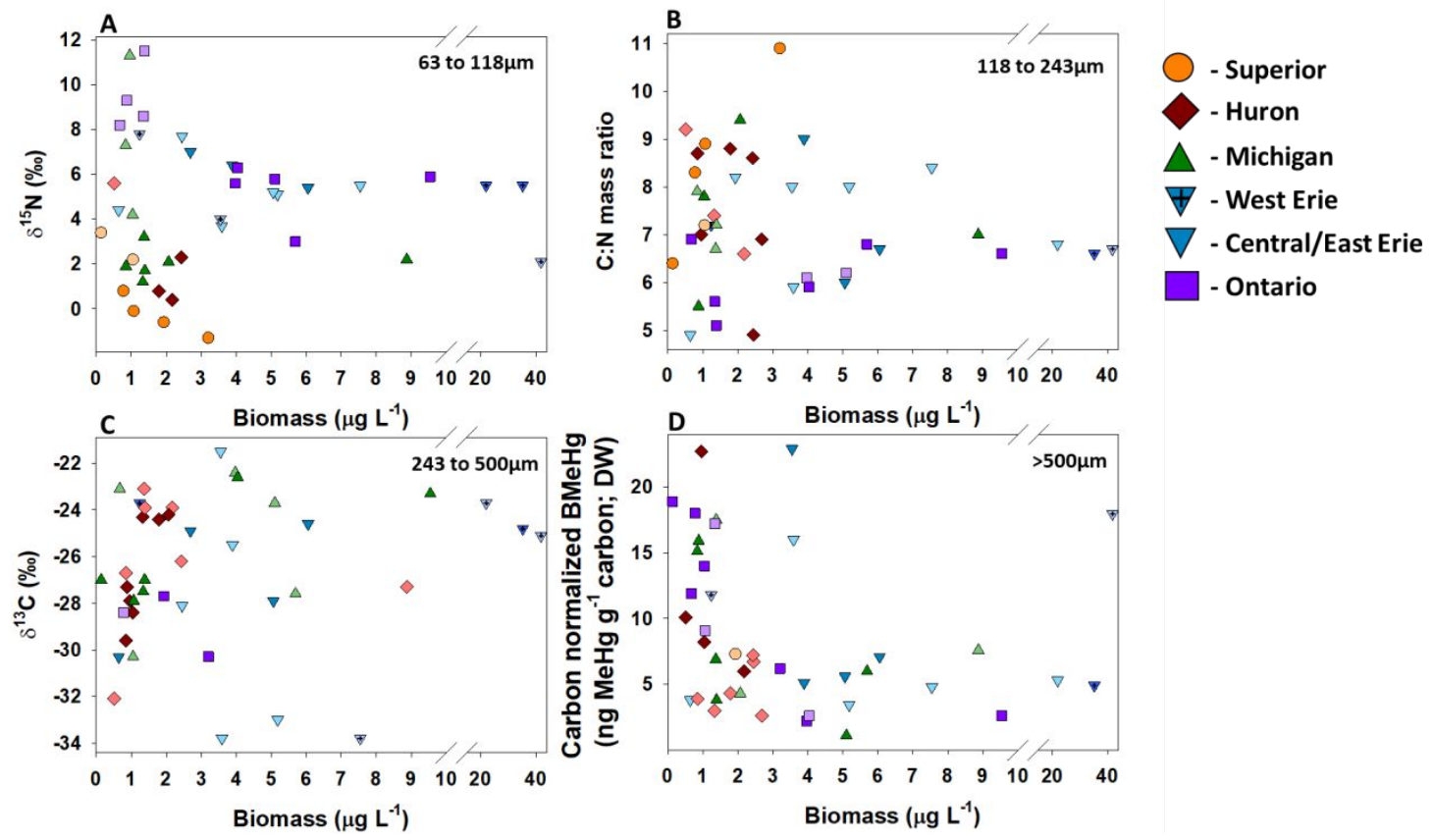
211 Figure S16. Physical and mercury concentration characterization of surficial $(0-2 \mathrm{~cm})$ sediments 212 in the Great Lakes. Percent sediment dry mass relative to percent sediment carbon (A), sediment 213 methylmercury (SMeHg) concentration relative to percent sediment carbon (B), percent of 214 sediment total mercury ( $\mathrm{SHgT}$ ) as $\mathrm{SMeHg}$ relative to percent sediment carbon (C), $\mathrm{SHgT}$ 215 concentration relative to percent sediment carbon (D), and SHgT concentration relative to percent 216 of $\mathrm{SHgT}$ as $\mathrm{SMeHg}$
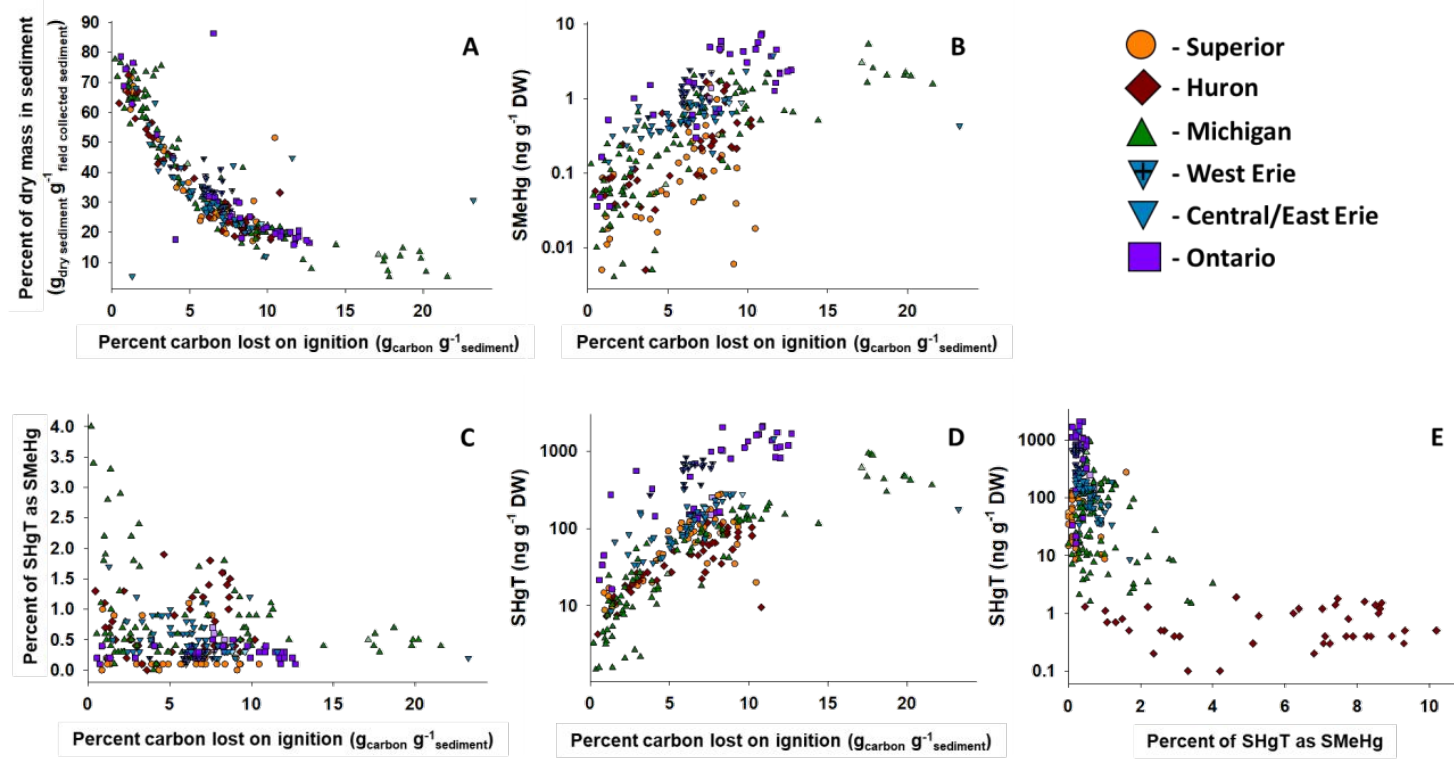
Figure S17. Mercury concentrations in composited mussel tissue (shells removed for all organisms $>5 \mathrm{~mm})$. Percent of total mercury $(\mathrm{BHgT})$ as methylmercury $(\mathrm{BMeHg})$ in mussel tissue relative to $\mathrm{BMeHg}$ concentration (A), percent of $\mathrm{BHgT}$ as $\mathrm{BMeHg}$ in mussel tissue relative to median shell length of sample composite (B), and BMeHg concentration in mussel tissue relative to median shell length of sample composite (C).
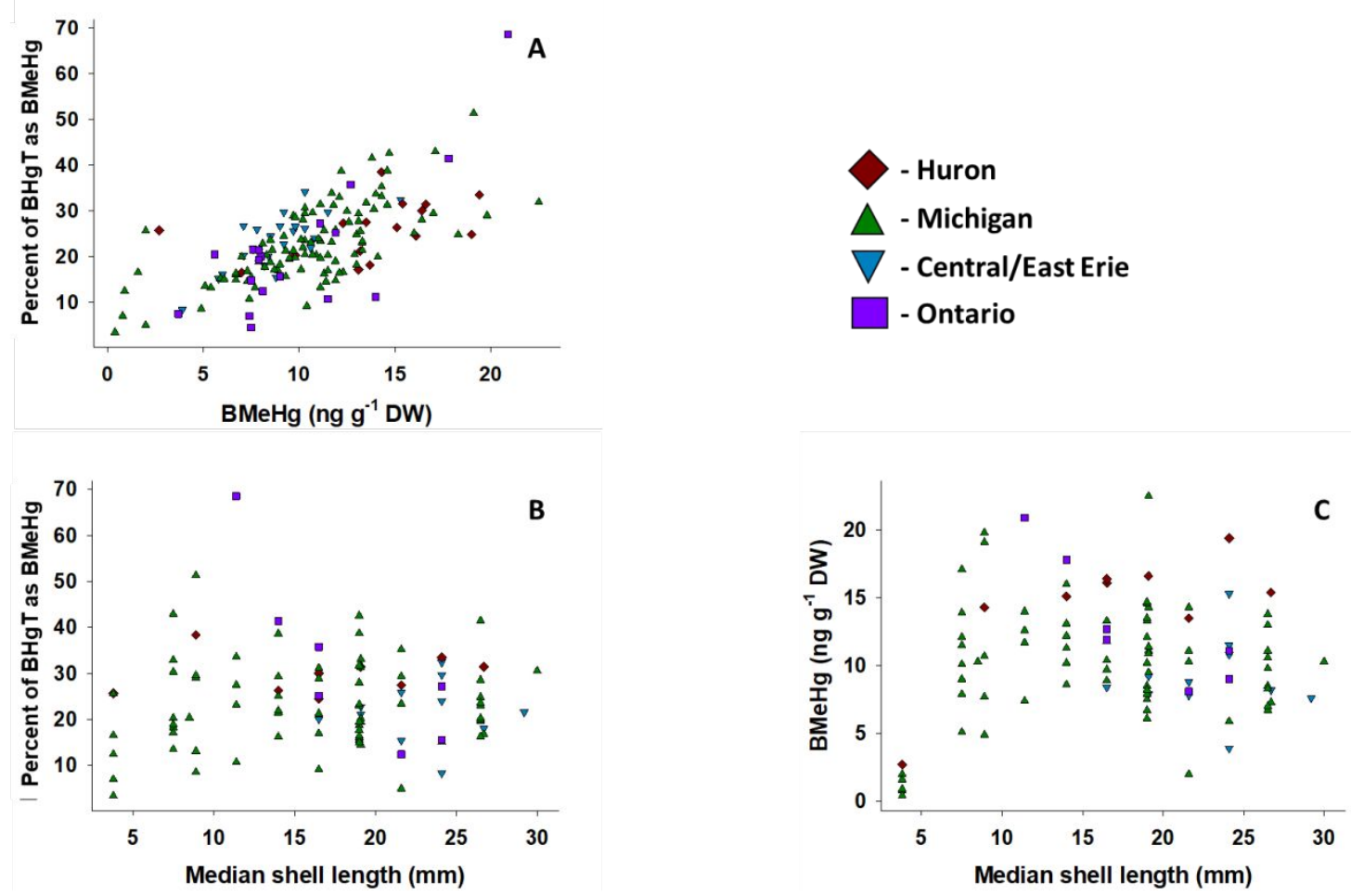

Figure S18. Specific ultraviolet absorbance at 254 nanometer wavelength $\left(\mathrm{SUVA}_{254 \mathrm{~nm}}\right)$ values for waters collected during the 2011 spring cruises. Paired with these are two dissolved organic matter isolates representing fulvic acids (FA) from dominantly autochthonous systems. ${ }^{4}$

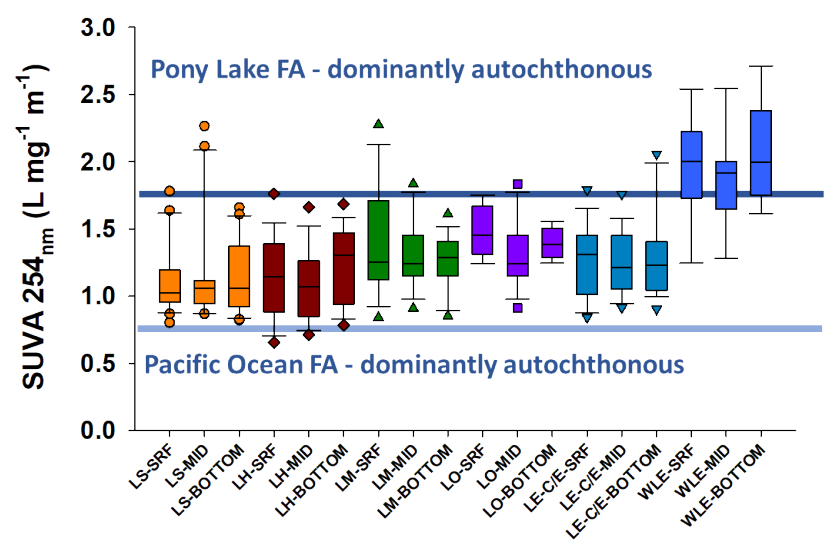


Table S1. Methodological details for the analysis of total mercury (HgT), methylmercury (MeHg), dissolved organic carbon (DOC), and the stable isotopes of carbon and nitrogen. Quality assurance acceptance criteria reported for analytical methods and field measurements (in parenthesis)

\begin{tabular}{|c|c|c|c|c|c|c|}
\hline $\begin{array}{l}\text { Analyte, Matrix, and } \\
\text { Method }\end{array}$ & $\begin{array}{l}\text { Quality Control } \\
\text { Objective }\end{array}$ & $\begin{array}{l}\text { Acceptance } \\
\text { Criteria } \\
\text { (Result) }\end{array}$ & Description & Frequency & Method Reference & Instrumentation \\
\hline \multirow[t]{6}{*}{$\begin{array}{c}\mathrm{HgT} \text { in Water by Atomic } \\
\text { Florescence }\end{array}$} & $\begin{array}{l}\text { Instrument Calibration } \\
\text { Linearity }\end{array}$ & $>0.995$ & $\begin{array}{l}\text { The } r^{2} \text { value for the slope of } \\
\text { the instrument calibration. }\end{array}$ & $\begin{array}{l}\text { At } \\
\text { instrument } \\
\text { calibration }\end{array}$ & $\begin{array}{c}\text { EPA Method 1631, Revision E: } \\
\text { Mercury in Water by } \\
\text { Oxidation, Purge and Trap, and } \\
\text { Cold Vapor Atomic } \\
\text { Fluorescence Spectrometry } \\
\end{array}$ & Tekran 2500 \\
\hline & $\begin{array}{l}\text { Instrument Calibration } \\
\text { Check Standard }\end{array}$ & $90-110 \%$ & $\begin{array}{l}\text { Percent recovery for the } \\
\text { analysis of a HgT standard } \\
\text { during sample analysis. }\end{array}$ & $\begin{array}{l}\text { Once every } \\
10 \text { samples }\end{array}$ & & \\
\hline & Instrument Duplicate & $<10 \%$ & $\begin{array}{l}\text { Percent difference of } \mathrm{HgT} \\
\text { concentration for duplicate } \\
\text { sample analysis. }\end{array}$ & All samples & & \\
\hline & Analytical Matrix Spike & $90-110 \%$ & $\begin{array}{c}\text { Percent recovery of } \mathrm{HgT} \\
\text { addition to a sample during } \\
\text { analysis. }\end{array}$ & $\begin{array}{l}\text { Once every } \\
10 \text { samples }\end{array}$ & & \\
\hline & Daily detection Limit & $<0.04 \mathrm{ng} / \mathrm{L}$ & $\begin{array}{l}\text { Three times the standard } \\
\text { deviation of the calibration } \\
\text { blanks. }\end{array}$ & $\begin{array}{l}\text { Determined } \\
\text { at instrument } \\
\text { calibration }\end{array}$ & & \\
\hline & Field Replicate & $\begin{array}{c}(20 \% \pm 17 \% \\
96)\end{array}$ & $\begin{array}{l}\text { Mean percent difference } \pm \\
\text { standard deviation, count. }\end{array}$ & & & \\
\hline
\end{tabular}




\begin{tabular}{|c|c|c|c|c|c|c|}
\hline $\begin{array}{l}\text { Analyte, Matrix, and } \\
\text { Method }\end{array}$ & $\begin{array}{l}\text { Quality Control } \\
\text { Objective }\end{array}$ & $\begin{array}{l}\text { Acceptance } \\
\text { Criteria } \\
\text { (Result) }\end{array}$ & Description & Frequency & Method Reference & Instrumentation \\
\hline \multirow[t]{7}{*}{$\begin{array}{l}\mathrm{HgT} \text { in Particulate Matter by } \\
\text { Atomic Florescence }\end{array}$} & $\begin{array}{c}\text { Instrument Calibration } \\
\text { Linearity }\end{array}$ & $>0.995$ & $\begin{array}{l}\text { The } r^{2} \text { value for the slope of } \\
\text { the instrument calibration. }\end{array}$ & $\begin{array}{c}\text { At } \\
\text { instrument } \\
\text { calibration }\end{array}$ & $\begin{array}{c}\text { USGS Techniques and } \\
\text { Methods 5A-8 } \\
\text { (https://pubs.usgs.gov/tm/2005/ } \\
\underline{\text { tm5A8/) }} \\
\text { EPA Method 1631, Revision E: } \\
\text { Mercury in Water by } \\
\text { Oxidation, Purge and Trap, and } \\
\text { Cold Vapor Atomic } \\
\text { Fluorescence Spectrometry }\end{array}$ & $\begin{array}{c}\text { Brooks Rand MERX-T } \\
\text { Automated Total Mercury } \\
\text { System }\end{array}$ \\
\hline & $\begin{array}{l}\text { Instrument Calibration } \\
\text { Check Standard }\end{array}$ & $90-110 \%$ & $\begin{array}{l}\text { Percent recovery for the } \\
\text { analysis of a HgT standard } \\
\text { during sample analysis. }\end{array}$ & $\begin{array}{l}\text { Once every } \\
10 \text { samples }\end{array}$ & & \\
\hline & Instrument Duplicate & $<10 \%$ & $\begin{array}{l}\text { Percent difference of } \mathrm{HgT} \\
\text { concentration for duplicate } \\
\text { analysis of a single sample } \\
\text { extract. }\end{array}$ & $\begin{array}{l}\text { Once every } \\
10 \text { samples }\end{array}$ & & \\
\hline & Analytical Matrix Spike & $85-115 \%$ & $\begin{array}{c}\text { Percent recovery of } \mathrm{HgT} \\
\text { addition to a sample during } \\
\text { analysis. }\end{array}$ & $\begin{array}{l}\text { Once every } \\
10 \text { samples }\end{array}$ & & \\
\hline & Detection Limit & N/A & $\begin{array}{c}\text { Determined as three times the } \\
\text { standard deviation of method } \\
\text { blanks for the analysis batch. } \\
\text { Results less than detection } \\
\text { limit not reported. }\end{array}$ & $\begin{array}{l}\text { Once per } \\
\text { setup batch }\end{array}$ & & \\
\hline & $\begin{array}{c}\text { Certified Reference } \\
\text { Material }\end{array}$ & $80-120 \%$ & $\begin{array}{l}\text { Certified Reference Material } \\
\text { prepared and analyzed similar } \\
\text { to samples. }\end{array}$ & $\begin{array}{l}\text { Once every } \\
10 \text { samples }\end{array}$ & & \\
\hline & Field Replicate & $\begin{array}{c}(17 \% \pm 25 \% \\
13)\end{array}$ & $\begin{array}{l}\text { Mean percent difference } \pm \\
\text { standard deviation, count. }\end{array}$ & & & \\
\hline
\end{tabular}




\begin{tabular}{|c|c|c|c|c|c|c|}
\hline $\begin{array}{l}\text { Analyte, Matrix, and } \\
\text { Method }\end{array}$ & $\begin{array}{l}\text { Quality Control } \\
\text { Objective }\end{array}$ & $\begin{array}{l}\text { Acceptance } \\
\text { Criteria } \\
\text { (Result) }\end{array}$ & Description & Frequency & Method Reference & Instrumentation \\
\hline \multirow[t]{8}{*}{$\begin{array}{l}\mathrm{HgT} \text { in Biota by Atomic } \\
\text { Florescence }\end{array}$} & $\begin{array}{c}\text { Instrument Calibration } \\
\text { Linearity }\end{array}$ & $>0.995$ & $\begin{array}{l}\text { The } r^{2} \text { value for the slope of } \\
\text { the instrument calibration. }\end{array}$ & $\begin{array}{c}\text { At } \\
\text { instrument } \\
\text { calibration }\end{array}$ & $\begin{array}{c}\text { Hammerschmidt, Chad R., and } \\
\text { William F. Fitzgerald. } \\
\text { "Bioaccumulation and trophic } \\
\text { transfer of methylmercury in } \\
\text { Long Island Sound." Archives } \\
\text { of Environmental } \\
\text { Contamination and } \\
\text { Toxicology } 51.3(2006): 416- \\
424 .^{6} \\
\text { EPA Method 1631, Revision E: } \\
\text { Mercury in Water by } \\
\text { Oxidation, Purge and Trap, and } \\
\text { Cold Vapor Atomic } \\
\text { Fluorescence Spectrometry }\end{array}$ & $\begin{array}{c}\text { Brooks Rand MERX-T } \\
\text { Automated Total Mercury } \\
\text { System }\end{array}$ \\
\hline & $\begin{array}{l}\text { Instrument Calibration } \\
\text { Check Standard }\end{array}$ & $90-110 \%$ & $\begin{array}{l}\text { Percent recovery for the } \\
\text { analysis of a HgT standard } \\
\text { during sample analysis. }\end{array}$ & $\begin{array}{l}\text { Once every } \\
10 \text { samples }\end{array}$ & & \\
\hline & Instrument Duplicate & $<10 \%$ & $\begin{array}{c}\text { Percent difference of } \mathrm{HgT} \\
\text { concentration for duplicate } \\
\text { analysis of a single sample } \\
\text { extract. }\end{array}$ & $\begin{array}{l}\text { Once every } \\
10 \text { samples }\end{array}$ & & \\
\hline & Method Triplicate & $<15 \%$ & $\begin{array}{l}\text { Relative standard deviation of } \\
\text { HgT concentration for } \\
\text { triplicate analysis of a sample. }\end{array}$ & $\begin{array}{l}\text { Once every } \\
15 \text { samples }\end{array}$ & & \\
\hline & Analytical Matrix Spike & $85-115 \%$ & $\begin{array}{c}\text { Percent recovery of } \mathrm{HgT} \\
\text { addition to a sample during } \\
\text { analysis. }\end{array}$ & $\begin{array}{l}\text { Once every } \\
10 \text { samples }\end{array}$ & & \\
\hline & Detection Limit & $\mathrm{N} / \mathrm{A}$ & $\begin{array}{c}\text { Determined as three times the } \\
\text { standard deviation of method } \\
\text { blanks for the analysis batch. } \\
\text { Results less than detection } \\
\text { limit not reported. }\end{array}$ & $\begin{array}{l}\text { Once per } \\
\text { setup batch }\end{array}$ & & \\
\hline & $\begin{array}{c}\text { Certified Reference } \\
\text { Material }\end{array}$ & $80-120 \%$ & $\begin{array}{l}\text { Certified Reference Material } \\
\text { prepared and analyzed similar } \\
\text { to samples. }\end{array}$ & $\begin{array}{l}\text { Once every } \\
10 \text { samples }\end{array}$ & & \\
\hline & Field Replicate & $\begin{array}{c}(26 \% \pm 35 \% \\
95)\end{array}$ & $\begin{array}{l}\text { Mean percent difference } \pm \\
\text { standard deviation, count. }\end{array}$ & & & \\
\hline
\end{tabular}




\begin{tabular}{|c|c|c|c|c|c|c|}
\hline $\begin{array}{c}\text { Analyte, Matrix, and } \\
\text { Method }\end{array}$ & $\begin{array}{c}\text { Quality Control } \\
\text { Objective }\end{array}$ & $\begin{array}{c}\text { Acceptance Criteria } \\
\text { (Result) }\end{array}$ & Description & Frequency & Method Reference & Instrumentation \\
\hline \multirow[t]{6}{*}{$\begin{array}{l}\text { HgT in Sediment by Direct } \\
\text { Combustion }\end{array}$} & $\begin{array}{c}\text { Instrument Calibration } \\
\text { Linearity }\end{array}$ & $>0.995$ & $\begin{array}{l}\text { The } r^{2} \text { value for the } \\
\text { slope of the instrument } \\
\text { calibration. }\end{array}$ & $\begin{array}{c}\text { At } \\
\text { instrument } \\
\text { calibration }\end{array}$ & $\begin{array}{l}\text { EPA Method } 7473 \text { (SW-846) } \\
\text { Rev. } 0 \text { - Direct Combustion }{ }^{2}\end{array}$ & Nippon MA-2000 \\
\hline & $\begin{array}{l}\text { Instrument Calibration } \\
\text { Check Standard }\end{array}$ & $90-110 \%$ & $\begin{array}{l}\text { Percent recovery for the } \\
\text { analysis of a } \mathrm{HgT} \\
\text { standard during sample } \\
\text { analysis. }\end{array}$ & $\begin{array}{l}\text { Two per } \\
\text { analysis } \\
\text { batch }\end{array}$ & & \\
\hline & Method Triplicate & $<15 \%$ & $\begin{array}{c}\text { Relative standard } \\
\text { deviation of } \mathrm{HgT} \\
\text { concentration for } \\
\text { triplicate analysis of a } \\
\text { sample. } \\
\end{array}$ & $\begin{array}{l}\text { Once every } \\
10 \text { samples }\end{array}$ & & \\
\hline & $\begin{array}{c}\text { Certified Reference } \\
\text { Material }\end{array}$ & $80-120 \%$ & $\begin{array}{l}\text { Percent recovery for a } \\
\text { certified reference } \\
\text { material prepared and } \\
\text { analyzed similar to } \\
\text { samples. }\end{array}$ & $\begin{array}{l}\text { Once every } \\
10 \text { samples }\end{array}$ & & \\
\hline & Detection Limit & $\mathrm{N} / \mathrm{A}$ & $\begin{array}{l}\text { Determined as three } \\
\text { times the standard } \\
\text { deviation of method } \\
\text { blanks for the analysis } \\
\text { batch. Results less than } \\
\text { detection limit not } \\
\text { reported. }\end{array}$ & $\begin{array}{l}\text { Once per } \\
\text { analytical } \\
\text { batch }\end{array}$ & & \\
\hline & Field Replicate & $(10 \% \pm 14 \%, 8)$ & $\begin{array}{c}\text { Mean percent difference } \\
\pm \text { standard deviation, } \\
\text { count. }\end{array}$ & & & \\
\hline
\end{tabular}


Table S1, continued.

\begin{tabular}{|c|c|c|c|c|c|c|}
\hline $\begin{array}{l}\text { Analyte, Matrix, and } \\
\text { Method }\end{array}$ & $\begin{array}{l}\text { Quality Control } \\
\text { Objective }\end{array}$ & $\begin{array}{c}\text { Acceptance Criteria } \\
\text { (Result) }\end{array}$ & Description & Frequency & Method Reference & Instrumentation \\
\hline \multirow[t]{6}{*}{$\begin{array}{l}\text { MeHg in Biota by Atomic } \\
\text { Florescence }\end{array}$} & $\begin{array}{c}\text { Instrument Calibration } \\
\text { Linearity }\end{array}$ & $>0.995$ & $\begin{array}{l}\text { The } r^{2} \text { value for the } \\
\text { slope of the instrument } \\
\text { calibration. }\end{array}$ & $\begin{array}{c}\text { At } \\
\text { instrument } \\
\text { calibration }\end{array}$ & $\begin{array}{c}\text { Hammerschmidt, Chad R., and } \\
\text { William F. Fitzgerald. } \\
\text { "Bioaccumulation and trophic } \\
\text { transfer of methylmercury in } \\
\text { Long Island Sound." Archives } \\
\text { of Environmental } \\
\text { Contamination and } \\
\text { Toxicology } 51.3(2006): 416- \\
424 .{ }^{6}\end{array}$ & $\begin{array}{l}\text { Brooks Rand MERX-M } \\
\text { Automated } \\
\text { Methylmercury System }\end{array}$ \\
\hline & $\begin{array}{l}\text { Instrument Calibration } \\
\text { Check Standard }\end{array}$ & $85-115 \%$ & $\begin{array}{c}\text { Percent recovery for } \\
\text { the analysis of a MeHg } \\
\text { standard during sample } \\
\text { analysis. }\end{array}$ & $\begin{array}{l}\text { Once every } \\
10 \text { samples }\end{array}$ & & \\
\hline & Method Triplicate & $<15 \%$ & $\begin{array}{l}\text { Relative standard } \\
\text { deviation of MeHg } \\
\text { concentration for } \\
\text { triplicate analysis of a } \\
\text { sample. } \\
\end{array}$ & $\begin{array}{l}\text { Once every } \\
15 \text { samples }\end{array}$ & & \\
\hline & Detection Limit & $\mathrm{N} / \mathrm{A}$ & $\begin{array}{l}\text { Determined as three } \\
\text { times the standard } \\
\text { deviation of method } \\
\text { blanks for the analysis } \\
\text { batch. Results less than } \\
\text { detection limit not } \\
\text { reported. }\end{array}$ & $\begin{array}{l}\text { Once per } \\
\text { setup batch }\end{array}$ & & \\
\hline & $\begin{array}{l}\text { Certified Reference } \\
\text { Material }\end{array}$ & $80-120 \%$ & $\begin{array}{l}\text { Certified Reference } \\
\text { Material prepared and } \\
\text { analyzed similar to } \\
\text { samples. }\end{array}$ & $\begin{array}{l}\text { Once every } \\
10 \text { samples }\end{array}$ & & \\
\hline & Field Replicate & $(23 \% \pm 27 \%, 95)$ & $\begin{array}{c}\text { Mean percent } \\
\text { difference } \pm \text { standard } \\
\text { deviation, count. }\end{array}$ & & & \\
\hline
\end{tabular}




\begin{tabular}{|c|c|c|c|c|c|c|}
\hline $\begin{array}{c}\text { Analyte, Matrix, and } \\
\text { Method }\end{array}$ & $\begin{array}{c}\text { Quality Control } \\
\text { Objective }\end{array}$ & $\begin{array}{c}\text { Acceptance Criteria } \\
\text { (Result) }\end{array}$ & Description & Frequency & Method Reference & Instrumentation \\
\hline \multirow[t]{6}{*}{$\begin{array}{c}\text { MeHg in Water by } \\
\text { Distillation and Isotope } \\
\text { Dilution }\end{array}$} & Mass Bias Correction & $<5 \%$ & $\begin{array}{c}\text { Correction for } \\
\text { instrument mass bias } \\
\text { for the isotope used for } \\
\text { dilution }\left(\mathrm{Me}^{199} \mathrm{Hg}\right) \\
\text { relative to } \mathrm{Me}^{202} \mathrm{Hg} \text {. } \\
\text { Relative standard } \\
\text { deviation for five } \\
\text { analyses. }\end{array}$ & $\begin{array}{l}\text { At } \\
\text { instrument } \\
\text { calibration }\end{array}$ & $\begin{array}{c}\text { USGS open file report 01-445 } \\
\text { (https://pubs.usgs.gov/of/2001/ } \\
\text { ofr-01-445/) } \\
\\
\text { EPA Method 1630: Methyl } \\
\text { Mercury in Water by } \\
\text { Distillatioin, Aqueous } \\
\text { Ethylatioin, Purge and Trap, } \\
\text { and Cold Vapor Atomic } \\
\text { Fluorescence Spectrometry }\end{array}$ & $\begin{array}{c}\text { Brooks Rand MERX-M } \\
\text { Automated } \\
\text { Methylmercury System } \\
\text { Perkin Elmer Elan } 9000 \\
\text { (before 2015) } \\
\\
\text { Thermo Scientific iCAP } \\
\text { RQ (after 2015) }\end{array}$ \\
\hline & $\begin{array}{l}\text { Isotope Dilution } \\
\text { Calibration }\end{array}$ & $<5 \%$ & $\begin{array}{l}\text { Calibration of mass of } \\
\text { isotope addition to } \\
\text { samples (Me } \mathrm{e}^{199} \mathrm{Hg} \text { or } \\
\mathrm{Me}^{204} \mathrm{Hg} \text { ). Relative } \\
\text { standard deviation for } \\
\text { five analyses. }\end{array}$ & $\begin{array}{c}\text { At } \\
\text { instrument } \\
\text { calibration }\end{array}$ & & \\
\hline & $\begin{array}{l}\text { Instrument Calibration } \\
\text { Check Standard }\end{array}$ & $85-115 \%$ & $\begin{array}{l}\text { Percent recovery for } \\
\text { the analysis of a MeHg } \\
\text { standard by isotope } \\
\text { dilution during sample } \\
\text { analysis. }\end{array}$ & $\begin{array}{c}\text { Once every } 6 \\
\text { samples }\end{array}$ & & \\
\hline & Analytical Matrix Spike & $75-125 \%$ & $\begin{array}{l}\text { Percent recovery of a } \\
\text { MeHg addition to a } \\
\text { sample during } \\
\text { distillation. } \\
\end{array}$ & $\begin{array}{l}\text { Once every } \\
10 \text { samples }\end{array}$ & & \\
\hline & Method Detection Limit & $(0.01 \mathrm{ng} / \mathrm{L})$ & $\begin{array}{l}\text { Determined as three } \\
\text { times the standard } \\
\text { deviation of replicate } \\
\text { sample analysis. }\end{array}$ & & & \\
\hline & Field Replicate & $(94 \% \pm 50 \%, 55)$ & $\begin{array}{c}\text { Mean percent } \\
\text { difference } \pm \text { standard } \\
\text { deviation, count. }\end{array}$ & & & \\
\hline
\end{tabular}




\begin{tabular}{|c|c|c|c|c|c|c|}
\hline $\begin{array}{c}\text { Analyte, Matrix, and } \\
\text { Method }\end{array}$ & $\begin{array}{c}\text { Quality Control } \\
\text { Objective }\end{array}$ & $\begin{array}{c}\text { Acceptance Criteria } \\
\text { (Result) }\end{array}$ & Description & Frequency & Method Reference & Instrumentation \\
\hline \multirow[t]{7}{*}{$\begin{array}{l}\text { MeHg in Water by Direct } \\
\text { Analysis and Isotope Dilution }\end{array}$} & Mass Bias Correction & $<5 \%$ & $\begin{array}{l}\text { Correction for } \\
\text { instrument mass bias } \\
\text { for the isotope used for } \\
\text { dilution }\left(\mathrm{Me}^{204} \mathrm{Hg}\right) \\
\text { relative to } \mathrm{Me}^{202} \mathrm{Hg} \text {. } \\
\text { Relative standard } \\
\text { deviation for five } \\
\text { analyses. }\end{array}$ & $\begin{array}{c}\text { At } \\
\text { instrument } \\
\text { calibration }\end{array}$ & $\begin{array}{c}\text { Lepak, Ryan F., et al. } \\
\text { "Influence of Cladophora- } \\
\text { quagga mussel assemblages on } \\
\text { nearshore methylmercury } \\
\text { production in lake } \\
\text { Michigan." Environmental } \\
\text { Science \& Technology 49.13 } \\
\text { (2015): 7606-7613." } \\
\text { Mansfield, Christopher R., and } \\
\text { Frank J. Black. "Quantification } \\
\text { of monomethylmercury in } \\
\text { natural waters by direct } \\
\text { ethylation: Interference } \\
\text { characterization and method } \\
\text { optimization." Limnology and } \\
\text { Oceanography: Methods } 13.2 \\
\text { (2015): 81-91. }\end{array}$ & $\begin{array}{c}\text { Brooks Rand MERX-M } \\
\text { Automated } \\
\text { Methylmercury System } \\
\text { Perkin Elmer Elan } 9000 \\
\text { (before 2015) } \\
\\
\text { Thermo Scientific iCAP } \\
\text { RQ (after 2015) }\end{array}$ \\
\hline & $\begin{array}{l}\text { Isotope Dilution } \\
\text { Calibration }\end{array}$ & $<5 \%$ & $\begin{array}{l}\text { Calibration of mass of } \\
\text { isotope addition to } \\
\text { samples }\left(\mathrm{Me}^{204} \mathrm{Hg}\right) \text {. } \\
\text { Relative standard } \\
\text { deviation for five } \\
\text { analyses. }\end{array}$ & $\begin{array}{l}\text { At } \\
\text { instrument } \\
\text { calibration }\end{array}$ & & \\
\hline & $\begin{array}{l}\text { Instrument Calibration } \\
\text { Check Standard }\end{array}$ & $(101 \% \pm 12 \%, 79)$ & $\begin{array}{l}\text { Average percent } \\
\text { recovery } \pm \text { standard } \\
\text { deviation, count. }\end{array}$ & $\begin{array}{c}\text { Once every } 6 \\
\text { samples }\end{array}$ & & \\
\hline & Field Spike & $(106 \% \pm 14 \%, 44)$ & $\begin{array}{l}\text { Average percent } \\
\text { recovery } \pm \text { standard } \\
\text { deviation, count. }\end{array}$ & & & \\
\hline & Method Detection Limit & $(0.002 \mathrm{ng} / \mathrm{L})$ & $\begin{array}{c}\text { Determined as three } \\
\text { times the standard } \\
\text { deviation of replicate } \\
\text { sample analysis } \\
(\mathrm{n}=20) .\end{array}$ & & & \\
\hline & Field Replicate & $(20 \% \pm 26 \%, 26)$ & $\begin{array}{c}\text { Mean percent } \\
\text { difference } \pm \text { standard } \\
\text { deviation, count. }\end{array}$ & & & \\
\hline & Field Blank & $(0.0022 \mathrm{ng} / \mathrm{L})$ & $\begin{array}{l}\text { Analysis of field- } \\
\text { collected and } \\
\text { processed blank water }\end{array}$ & 20 & & \\
\hline
\end{tabular}




\begin{tabular}{|c|c|c|c|c|c|c|}
\hline $\begin{array}{c}\text { Analyte, Matrix, and } \\
\text { Method }\end{array}$ & $\begin{array}{l}\text { Quality Control } \\
\text { Objective }\end{array}$ & $\begin{array}{l}\text { Acceptance } \\
\text { Criteria } \\
\text { (Result) }\end{array}$ & Description & Frequency & Method Reference & Instrumentation \\
\hline \multirow[t]{8}{*}{$\begin{array}{l}\text { MeHg in Particulate } \\
\text { Matter by Distillation } \\
\text { and Isotope Dilution }\end{array}$} & Mass Bias Correction & $<5 \%$ & $\begin{array}{l}\text { Correction for instrument mass } \\
\text { bias for the isotope used for } \\
\text { dilution }\left(\mathrm{Me}{ }^{199} \mathrm{Hg}\right) \text { relative to } \\
\mathrm{Me}^{202} \mathrm{Hg} \text {. Relative standard } \\
\text { deviation for five analyses. }\end{array}$ & $\begin{array}{c}\text { At } \\
\text { instrument } \\
\text { calibration }\end{array}$ & $\begin{array}{c}\text { USGS Techniques and } \\
\text { Methods 5A-7 } \\
\text { (https://pubs.usgs.gov/tm/2005/ } \\
\text { tm5A7/) } \\
\text { EPA Method 1630: Methyl } \\
\text { Mercury in Water by } \\
\text { Distillatioin, Aqueous } \\
\text { Ethylatioin, Purge and Trap, } \\
\text { and Cold Vapor Atomic } \\
\text { Fluorescence Spectrometry }{ }^{1}\end{array}$ & $\begin{array}{c}\text { Brooks Rand MERX-M } \\
\text { Automated } \\
\text { Methylmercury System } \\
\text { Perkin Elmer Elan } 9000 \\
\text { (before 2015) } \\
\\
\text { Thermo Scientific iCAP } \\
\text { RQ (after 2015) }\end{array}$ \\
\hline & $\begin{array}{l}\text { Isotope Dilution } \\
\text { Calibration }\end{array}$ & $<5 \%$ & $\begin{array}{l}\text { Calibration of mass of isotope } \\
\text { addition to samples }\left(\mathrm{Me}^{199} \mathrm{Hg} \text { or }\right. \\
\mathrm{Me}^{204} \mathrm{Hg} \text { ). Relative standard } \\
\text { deviation for five analyses. }\end{array}$ & $\begin{array}{l}\text { At } \\
\text { instrument } \\
\text { calibration }\end{array}$ & & \\
\hline & $\begin{array}{c}\text { Instrument Calibration } \\
\text { Check Standard }\end{array}$ & $85-115 \%$ & $\begin{array}{l}\text { Percent recovery for the analysis } \\
\text { of a MeHg standard by isotope } \\
\text { dilution during sample analysis. }\end{array}$ & $\begin{array}{l}\text { Once every } \\
6 \text { samples }\end{array}$ & & \\
\hline & Mass Bias Correction & $<5 \%$ & $\begin{array}{c}\text { Correction for instrument mass } \\
\text { bias for isotope dilution addition } \\
\left(\mathrm{Me}^{199} \mathrm{Hg} \text { or } \mathrm{Me}^{204} \mathrm{Hg}\right) \text { relative to } \\
\mathrm{Me}^{202} \mathrm{Hg} \text {. Relative standard } \\
\text { deviation for five analyses. }\end{array}$ & $\begin{array}{c}\text { At } \\
\text { instrument } \\
\text { calibration }\end{array}$ & & \\
\hline & $\begin{array}{l}\text { Isotope Dilution } \\
\text { Calibration }\end{array}$ & $<5 \%$ & $\begin{array}{l}\text { Calibration of mass of isotope } \\
\text { addition to samples }\left(\mathrm{Me}^{199} \mathrm{Hg} \text { or }\right. \\
\mathrm{Me}^{204} \mathrm{Hg} \text { ). Relative standard } \\
\text { deviation for five analyses. }\end{array}$ & $\begin{array}{c}\text { At } \\
\text { instrument } \\
\text { calibration }\end{array}$ & & \\
\hline & $\begin{array}{c}\text { Certified Reference } \\
\text { Material }\end{array}$ & $75-125 \%$ & $\begin{array}{c}\text { Certified Reference Material } \\
\text { prepared and analyzed similar to } \\
\text { samples. }\end{array}$ & $\begin{array}{l}\text { Once every } \\
10 \text { samples }\end{array}$ & & \\
\hline & Detection Limit & N/A & $\begin{array}{l}\text { Determined as three times the } \\
\text { standard deviation of method } \\
\text { blanks for the analysis batch. } \\
\text { Results less than detection limit } \\
\text { not reported. }\end{array}$ & $\begin{array}{l}\text { Once per } \\
\text { analytical } \\
\text { batch }\end{array}$ & & \\
\hline & Field Replicate & $(25 \% \pm 27 \%, 20)$ & $\begin{array}{l}\text { Mean percent difference } \pm \\
\text { standard deviation, count. }\end{array}$ & & & \\
\hline
\end{tabular}




\begin{tabular}{|c|c|c|c|c|c|c|}
\hline $\begin{array}{c}\text { Analyte, Matrix, and } \\
\text { Method }\end{array}$ & $\begin{array}{l}\text { Quality Control } \\
\text { Objective }\end{array}$ & $\begin{array}{c}\text { Acceptance Criteria } \\
\text { (Result) }\end{array}$ & Description & Frequency & Method Reference & Instrumentation \\
\hline \multirow[t]{7}{*}{$\begin{array}{c}\text { MeHg in Sediment by } \\
\text { Distillation and Isotope } \\
\text { Dilution }\end{array}$} & Mass Bias Correction & $<5 \%$ & $\begin{array}{l}\text { Correction for } \\
\text { instrument mass bias } \\
\text { for the isotope used for } \\
\text { dilution }\left(\mathrm{Me}^{199} \mathrm{Hg}\right) \\
\text { relative to } \mathrm{Me}^{202} \mathrm{Hg} \text {. } \\
\text { Relative standard } \\
\text { deviation for five } \\
\text { analyses. }\end{array}$ & $\begin{array}{c}\text { At } \\
\text { instrument } \\
\text { calibration }\end{array}$ & $\begin{array}{c}\text { USGS Techniques and } \\
\text { Methods 5A-7 } \\
\text { (https://pubs.usgs.gov/tm/2005/ } \\
\text { tm5A7/) }\end{array}$ & $\begin{array}{l}\text { Brooks Rand MERX-M } \\
\text { Automated } \\
\text { Methylmercury System } \\
\text { Perkin Elmer Elan } 9000 \\
\text { (before 2015) } \\
\\
\text { Thermo Scientific iCAP } \\
\text { RQ (after 2015) }\end{array}$ \\
\hline & $\begin{array}{l}\text { Isotope Dilution } \\
\text { Calibration }\end{array}$ & $<5 \%$ & $\begin{array}{l}\text { Calibration of mass of } \\
\text { isotope addition to } \\
\text { samples }\left(\mathrm{Me}^{199} \mathrm{Hg} \text { or }\right. \\
\mathrm{Me}^{204} \mathrm{Hg} \text {. Relative } \\
\text { standard deviation for } \\
\text { five analyses. }\end{array}$ & $\begin{array}{c}\text { At } \\
\text { instrument } \\
\text { calibration }\end{array}$ & & \\
\hline & $\begin{array}{l}\text { Instrument Calibration } \\
\text { Check Standard }\end{array}$ & $85-115 \%$ & $\begin{array}{l}\text { Percent recovery for } \\
\text { the analysis of a MeHg } \\
\text { standard by isotope } \\
\text { dilution during sample } \\
\text { analysis. } \\
\end{array}$ & $\begin{array}{l}\text { Once every } 6 \\
\text { samples }\end{array}$ & & \\
\hline & Method Triplicate & $<25 \%$ & $\begin{array}{c}\text { Relative standard } \\
\text { deviation of MeHg } \\
\text { concentration for } \\
\text { triplicate analysis of a } \\
\text { sample. } \\
\end{array}$ & $\begin{array}{l}\text { Once every } \\
15 \text { samples }\end{array}$ & & \\
\hline & $\begin{array}{c}\text { Certified Reference } \\
\text { Material }\end{array}$ & $80-120 \%$ & $\begin{array}{l}\text { Certified Reference } \\
\text { Material prepared and } \\
\text { analyzed similar to } \\
\text { samples. }\end{array}$ & $\begin{array}{l}\text { Once every } \\
15 \text { samples }\end{array}$ & & \\
\hline & Detection Limit & N/A & $\begin{array}{c}\text { Determined as three } \\
\text { times the standard } \\
\text { deviation of method } \\
\text { blanks for the analysis } \\
\text { batch. Results less than } \\
\text { detection limit not } \\
\text { reported. }\end{array}$ & $\begin{array}{l}\text { Once per } \\
\text { analytical } \\
\text { batch }\end{array}$ & & \\
\hline & Field Replicate & $(17 \% \pm 11 \%, 6)$ & $\begin{array}{c}\text { Mean percent } \\
\text { difference } \pm \text { standard } \\
\text { deviation, count. }\end{array}$ & & & \\
\hline
\end{tabular}




\begin{tabular}{|c|c|c|c|c|c|c|}
\hline $\begin{array}{l}\text { Analyte, Matrix, and } \\
\text { Method }\end{array}$ & $\begin{array}{l}\text { Quality Control } \\
\text { Objective }\end{array}$ & $\begin{array}{c}\begin{array}{c}\text { Acceptance Criteria } \\
\text { (Result) }\end{array} \\
\text { (R) }\end{array}$ & Description & Frequency & Method Reference & Instrumentation \\
\hline \multirow[t]{5}{*}{$\begin{array}{c}\text { DOC in Water by Catalytic } \\
\text { Oxidation and Infrared } \\
\text { Detection }\end{array}$} & $\begin{array}{l}\text { Instrument Calibration } \\
\text { Linearity }\end{array}$ & $>0.995$ & $\begin{array}{l}\text { The } \mathrm{r}^{2} \text { value for the } \\
\text { slope of the instrument } \\
\text { calibration. }\end{array}$ & $\begin{array}{c}\text { At } \\
\text { instrument } \\
\text { calibration }\end{array}$ & $\begin{array}{l}\text { American Public Health } \\
\text { Association. "Standard } \\
\text { methods for the examination of } \\
\text { water and wastewater } 21 \mathrm{st} \\
\text { Edition Method } 5310 \text { B. High } \\
\text { temperature combustion } \\
\text { method." Standard Methods for } \\
\text { the Examination of Water and } \\
\text { Wastewater. APHA, AWWA, } \\
\text { WEF Washington, D.C., USA, } \\
\text { 2005. 5-21.9 }\end{array}$ & Shimadzu TOC-V $\mathrm{V}_{\mathrm{CSH}}$ \\
\hline & $\begin{array}{l}\text { Instrument Calibration } \\
\text { Check Standard }\end{array}$ & $90-110 \%$ & $\begin{array}{c}\text { Percent recovery for } \\
\text { the analysis of a DOC } \\
\text { standard during sample } \\
\text { analysis. }\end{array}$ & $\begin{array}{l}\text { Once every } \\
10 \text { samples }\end{array}$ & & \\
\hline & Instrument Duplicate & $<10 \%$ & $\begin{array}{l}\text { Percent difference of } \\
\text { DOC concentration for } \\
\text { duplicate sample } \\
\text { analysis. }\end{array}$ & $\begin{array}{l}\text { Once every } \\
10 \text { samples }\end{array}$ & & \\
\hline & Detection Limit & $(0.2 \mathrm{mg} / \mathrm{L})$ & $\begin{array}{l}\text { Determined as three } \\
\text { times the standard } \\
\text { deviation of field } \\
\text { blanks. Results less } \\
\text { than detection limit not } \\
\text { reported. }\end{array}$ & & & \\
\hline & Field Replicate & $(3 \% \pm 2 \%, 66)$ & $\begin{array}{c}\text { Mean percent } \\
\text { difference } \pm \text { standard } \\
\text { deviation, count. }\end{array}$ & & & \\
\hline
\end{tabular}


251 Table S1, continued.

\begin{tabular}{|c|c|c|c|c|c|c|}
\hline $\begin{array}{c}\text { Analyte, Matrix, and } \\
\text { Method }\end{array}$ & $\begin{array}{l}\text { Quality Control } \\
\text { Objective }\end{array}$ & $\begin{array}{c}\text { Acceptance Criteria } \\
\text { (Result) }\end{array}$ & Description & Frequency & Method Reference & Instrumentation \\
\hline \multirow[t]{4}{*}{$\begin{array}{c}\text { Stable Isotopes of Carbon and } \\
\text { Nitrogen in Biota }\end{array}$} & Analytical Error & $( \pm 0.1 \%)$ & $\begin{array}{l}\text { Analytical error as the } \\
\text { mean standard } \\
\text { deviation (SD) of } \\
\text { replicate analyses }\end{array}$ & $\begin{array}{l}\text { Once every } \\
10 \text { samples }\end{array}$ & $\begin{array}{l}\text { Sierszen, Michael E., et al. } \\
\text { "Depth gradients in food-web } \\
\text { processes linking habitats in } \\
\text { large lakes: Lake Superior as } \\
\text { an exemplar } \\
\text { ecosystem." Freshwater } \\
\text { Biology 59.10 (2014): 2122- } \\
2136 . .^{10} \\
\end{array}$ & $\begin{array}{c}\text { Costech } 4010 \text { Elemental } \\
\text { Analyzer } \\
\text { Thermo Delta Plus XP } \\
\text { Mass Spectrometer }\end{array}$ \\
\hline & Linearity & $\begin{array}{l}\text { (pseudo linearity } \\
\text { without mass bias, } \\
>0.5 \% \text { ) }\end{array}$ & $\begin{array}{c}\text { Multiple paired } \\
\text { analyses of lab } \\
\text { standards increasing in } \\
\text { mass are analyzed to } \\
\text { evaluate instrument. }\end{array}$ & $\begin{array}{c}\text { Once every } \\
\text { week or as } \\
\text { needed. }\end{array}$ & & \\
\hline & Accuracy & $\begin{array}{l}\text { (Within } 0.5 \% \text { of } \\
\text { long-term value) }\end{array}$ & $\begin{array}{l}\text { Compared to previous } \\
\text { long-term standards } \\
\text { that include lab- } \\
\text { produced or biological } \\
\text { character. }\end{array}$ & $\begin{array}{l}\text { Once every } \\
10 \text { samples }\end{array}$ & & \\
\hline & Blank contribution & $\begin{array}{c}(<0.1 \% \text { sample } \\
\text { voltage })\end{array}$ & $\begin{array}{l}\text { Measurement on gas } \\
\text { and empty tins to } \\
\text { evaluate leak and } \\
\text { contamination } \\
\text { potentials. }\end{array}$ & $\begin{array}{l}\text { To begin and } \\
\text { end every } \\
\text { analytical } \\
\text { sequence. }\end{array}$ & & \\
\hline
\end{tabular}


254 Table S2. Sampling site coordinates for Lake Erie recorded onboard the R/V Lake Guardian.

\begin{tabular}{|c|c|c|c|}
\hline Lake & Site name & Latitude & Longitude \\
\hline ERIE & ER02-GS & 41.52000 & -81.71800 \\
\hline ERIE & ER07-GS & 41.93000 & -80.79800 \\
\hline ERIE & ER09 & 42.53800 & -79.61700 \\
\hline ERIE & ER10 & 42.68000 & -79.69500 \\
\hline ERIE & ER100-GS & 42.35000 & -79.61500 \\
\hline ERIE & ER101-GS & 42.58000 & -79.15200 \\
\hline ERIE & ER102-GS & 42.68000 & -80.24800 \\
\hline ERIE & ER103-GS & 42.64000 & -81.16200 \\
\hline ERIE & ER104-GS & 42.18000 & -82.20600 \\
\hline ERIE & ER105-GS & 41.59000 & -82.90700 \\
\hline ERIE & ER106-GS & 41.48000 & -82.20100 \\
\hline ERIE & ER107-GS & 42.62000 & -80.80900 \\
\hline ERIE & ER108-GS & 41.94000 & -83.01000 \\
\hline ERIE & ER109-GS & 41.81000 & -83.16000 \\
\hline ERIE & ER15M & 42.51600 & -79.89200 \\
\hline ERIE & ER30 & 42.43000 & -81.20500 \\
\hline ERIE & ER31 & 42.25300 & -81.10600 \\
\hline ERIE & ER32 & 42.08200 & -81.01100 \\
\hline ERIE & ER36 & 41.93500 & -81.47900 \\
\hline ERIE & ER37 & 42.11000 & -81.57500 \\
\hline ERIE & ER38 & 42.28100 & -81.67200 \\
\hline ERIE & ER42 & 41.96400 & -82.04100 \\
\hline ERIE & ER43 & 41.78800 & -81.94500 \\
\hline ERIE & ER58 & 41.68600 & -81.06600 \\
\hline ERIE & ER59 & 41.72700 & -83.15000 \\
\hline ERIE & ER60 & 41.89200 & -83.19700 \\
\hline ERIE & ER61 & 41.94700 & -83.04400 \\
\hline ERIE & ER63 & 42.41700 & -79.80100 \\
\hline ERIE & ER73 & 41.97800 & -81.75700 \\
\hline ERIE & ER78M & 42.11700 & -81.25000 \\
\hline ERIE & ER82-GS & 42.46000 & -80.36000 \\
\hline ERIE & ER83-GS & 42.82000 & -79.58100 \\
\hline ERIE & ER86-GS & 42.86000 & -78.93200 \\
\hline ERIE & ER91M & 41.84000 & -82.91700 \\
\hline ERIE & ER92 & 41.95000 & -82.68600 \\
\hline ERIE & ER93B & 42.61640 & -80.00013 \\
\hline ERIE & ER95B & 42.00017 & -80.66684 \\
\hline ERIE & ERFE & 41.58280 & -82.91708 \\
\hline ERIE & ERFO & 42.41700 & -79.58400 \\
\hline
\end{tabular}


256 Table S3. Sampling site coordinates for Lake Huron recorded onboard the $R / V$ Lake Guardian.

\begin{tabular}{|c|c|c|c|}
\hline Lake & Site name & Latitude & Longitude \\
\hline HURON & HU06 & 43.46700 & -82.00000 \\
\hline HURON & HU09 & 43.63400 & -82.21800 \\
\hline HURON & HU12 & 43.89000 & -82.05800 \\
\hline HURON & HU15M & 44.00000 & -82.35000 \\
\hline HURON & HU27 & 44.19800 & -82.50400 \\
\hline HURON & HU32 & 44.45300 & -82.34200 \\
\hline HURON & HU37 & 44.76200 & -82.78300 \\
\hline HURON & HU38 & 44.74000 & -82.06000 \\
\hline HURON & HU45M & 45.13700 & -82.98300 \\
\hline HURON & HU48 & 45.27900 & -82.45200 \\
\hline HURON & HU53 & 45.45000 & -82.91600 \\
\hline HURON & HU54M & 45.51700 & -83.41600 \\
\hline HURON & HU61 & 45.75000 & -83.91700 \\
\hline HURON & HU93 & 44.10000 & -82.11700 \\
\hline HURON & HU95B & 44.33385 & -82.83280 \\
\hline HURON & HU96B & 44.58332 & -81.49985 \\
\hline HURON & HU97B & 44.91675 & -83.16557 \\
\hline HURON & HUFE & 45.25383 & -83.24952 \\
\hline HURON & HUFO & 44.08400 & -82.75100 \\
\hline & & & \\
\hline
\end{tabular}


258 Table S4. Sampling site coordinates for Lake Michigan recorded onboard the $R / V$ Lake Guardian.

\begin{tabular}{|c|c|c|c|}
\hline Lake & Site name & Latitude & Longitude \\
\hline MICHIGAN & MI100-GS & 43.01348 & -87.81125 \\
\hline MICHIGAN & MI101-GS & 42.12389 & -87.09238 \\
\hline MICHIGAN & MI102-GS & 41.88468 & -87.48983 \\
\hline MICHIGAN & MI103-GS & 41.68662 & -87.31276 \\
\hline MICHIGAN & MI104-GS & 41.68958 & -87.01699 \\
\hline MICHIGAN & MI105-GS & 42.11831 & -86.51593 \\
\hline MICHIGAN & MI106-GS & 42.67926 & -86.24049 \\
\hline MICHIGAN & MI107-GS & 43.06260 & -86.27747 \\
\hline MICHIGAN & MI108-GS & 43.09344 & -86.53285 \\
\hline MICHIGAN & MI109-GS & 43.22183 & -86.36486 \\
\hline MICHIGAN & MI11 & 42.38500 & -87.00100 \\
\hline MICHIGAN & MI110-GS & 43.63707 & -86.62220 \\
\hline MICHIGAN & MI111-GS & 43.95653 & -86.49435 \\
\hline MICHIGAN & MI112-GS & 44.75033 & -86.57979 \\
\hline MICHIGAN & MI113-GS & 44.96895 & -86.03704 \\
\hline MICHIGAN & MI114-GS & 45.60991 & -87.04531 \\
\hline MICHIGAN & MI115-GS & 45.09448 & -87.54638 \\
\hline MICHIGAN & MI116-GS & 44.80779 & -87.72636 \\
\hline MICHIGAN & MI117-GS & 44.06445 & -87.47346 \\
\hline MICHIGAN & MI118-GS & 44.13683 & -87.51739 \\
\hline MICHIGAN & MI119-GS & 44.08526 & -87.58883 \\
\hline MICHIGAN & MI120-GS & 43.74807 & -87.67042 \\
\hline MICHIGAN & MI17 & 42.73400 & -87.41500 \\
\hline MICHIGAN & MI18M & 42.73300 & -87.00100 \\
\hline MICHIGAN & MI19 & 42.73400 & -86.58400 \\
\hline MICHIGAN & MI23 & 43.13300 & -87.00000 \\
\hline MICHIGAN & MI27M & 43.60000 & -86.91700 \\
\hline MICHIGAN & MI30B & 43.93298 & -86.56703 \\
\hline MICHIGAN & MI31B & 43.91687 & -87.61607 \\
\hline MICHIGAN & MI32 & 44.14100 & -87.23300 \\
\hline MICHIGAN & MI34 & 44.09000 & -86.76700 \\
\hline MICHIGAN & MI40 & 44.75900 & -86.96600 \\
\hline MICHIGAN & MI41M & 44.73500 & -86.72200 \\
\hline MICHIGAN & MI42B & 44.77043 & -87.21268 \\
\hline MICHIGAN & MI46B & 43.10267 & -86.37087 \\
\hline MICHIGAN & MI47 & 45.17800 & -86.37500 \\
\hline MICHIGAN & MI48B & 42.68305 & -86.3333 \\
\hline MICHIGAN & MI49B & 45.49130 & -87.03301 \\
\hline MICHIGAN & MI50B & 45.11634 & -87.41583 \\
\hline MICHIGAN & MI51B & 45.18307 & -86.10078 \\
\hline MICHIGAN & MI52B & 45.80825 & -86.04558 \\
\hline MICHIGAN & MI53B & 45.43350 & -85.21615 \\
\hline MICHIGAN & MIFE & 42.58300 & -86.41700 \\
\hline MICHIGAN & MIFO & 44.75000 & -87.08300 \\
\hline
\end{tabular}

259 
260 Table S5. Sampling site coordinates for Lake Ontario recorded onboard the $R / V$ Lake Guardian.

\begin{tabular}{|c|c|c|c|}
\hline Lake & Site name & Latitude & Longitude \\
\hline ONTARIO & ON12 & 43.50300 & -79.35200 \\
\hline ONTARIO & ON25 & 43.51700 & -79.08000 \\
\hline ONTARIO & ON33M & 43.59600 & -78.80200 \\
\hline ONTARIO & ON41 & 43.71600 & -78.02700 \\
\hline ONTARIO & ON49 & 43.77200 & -77.43800 \\
\hline ONTARIO & ON55M & 43.44400 & -77.43800 \\
\hline ONTARIO & ON60 & 43.58000 & -77.19900 \\
\hline ONTARIO & ON63 & 43.73100 & -77.01700 \\
\hline ONTARIO & ON64B & 43.58333 & -76.33333 \\
\hline ONTARIO & ON65B & 43.30870 & -76.95053 \\
\hline ONTARIO & ON67B & 43.37477 & -78.72923 \\
\hline ONTARIO & ON68B & 43.58334 & -79.41667 \\
\hline ONTARIO & ON69B & 43.31845 & -79.00012 \\
\hline ONTARIO & ONFE & 43.58000 & -76.25000 \\
\hline ONTARIO & ONFO & 43.41650 & -77.91650 \\
\hline ONTARIO & CORNELL SITE 16 & 43.27000 & -79.35900 \\
\hline
\end{tabular}


262 Table S6. Sampling site coordinates for Lake Superior recorded onboard the R/V Lake Guardian.

\begin{tabular}{|c|c|c|c|}
\hline Lake & Site name & Latitude & Longitude \\
\hline SUPERIOR & SU01M & 46.99300 & -85.16000 \\
\hline SUPERIOR & SU02 & 47.36000 & -85.62000 \\
\hline SUPERIOR & SU03 & 46.89500 & -85.85200 \\
\hline SUPERIOR & SU04 & 47.25883 & -86.34933 \\
\hline SUPERIOR & SU05 & 46.77483 & -86.55567 \\
\hline SUPERIOR & SU06 & 48.55800 & -86.37567 \\
\hline SUPERIOR & SU07 & 48.07400 & -86.59148 \\
\hline SUPERIOR & SU08M & 47.60517 & -86.81783 \\
\hline SUPERIOR & SU09 & 48.43650 & -87.08600 \\
\hline SUPERIOR & SU10 & 47.51433 & -87.54633 \\
\hline SUPERIOR & SU11 & 48.34367 & -87.82450 \\
\hline SUPERIOR & SU12 & 47.85667 & -88.04167 \\
\hline SUPERIOR & SU13 & 48.24983 & -88.54367 \\
\hline SUPERIOR & SU14 & 47.74167 & -88.73800 \\
\hline SUPERIOR & SU15 & 48.08283 & -89.25267 \\
\hline SUPERIOR & SU16 & 47.62117 & -89.46333 \\
\hline SUPERIOR & SU17M & 47.16400 & -89.66250 \\
\hline SUPERIOR & SU18 & 47.51417 & -90.15167 \\
\hline SUPERIOR & SU19 & 47.37000 & -90.85400 \\
\hline SUPERIOR & SU20B & 46.88362 & -90.28273 \\
\hline SUPERIOR & SU21B & 47.15873 & -87.78635 \\
\hline SUPERIOR & SU22B & 46.79983 & -91.75020 \\
\hline SUPERIOR & SU23B & 46.59715 & -84.80660 \\
\hline SUPERIOR & SUFE & 46.91667 & -90.41558 \\
\hline SUPERIOR & SUFO & 47.41667 & -87.57850 \\
\hline
\end{tabular}

263 
264

Table S7. Average Laurentian Great Lakes methylmercury concentrations (SMeHg as ng/g dry weight), total mercury concentrations ( $\mathrm{SHgT}$ as ng/g dry weight), and percent loss on ignition (LOI) in surficial sediments (0-2 cm) collected in August and September (2010-2014) onboard the $R / V$ Lake Guardian. Variability was measured across all samples collected as standard deviation (when $n>2$ ) or as a range of values (when $\mathrm{n}=2$, in parenthesis).

\begin{tabular}{|c|c|c|c|c|c|c|c|c|c|}
\hline Site Name & SMeHg & $\begin{array}{c}\text { Standard } \\
\text { Deviation } \\
\text { (range) }\end{array}$ & $\mathbf{n}$ & SHgT & $\begin{array}{c}\text { Standard } \\
\text { Deviation } \\
\text { (range) }\end{array}$ & $\mathbf{n}$ & LOI & $\begin{array}{c}\text { Standard } \\
\text { Deviation } \\
\text { (range) }\end{array}$ & $\mathbf{n}$ \\
\hline ER02-GS & 0.319 & & 1 & 67.0 & & 1 & 1.54 & & 1 \\
\hline ER07-GS & 0.390 & & 1 & 46.0 & & 1 & 2.38 & & 1 \\
\hline ER101-GS & 0.117 & & 1 & 34.0 & & 1 & 2.77 & & 1 \\
\hline ER102-GS & 0.142 & & 1 & 8.50 & & 1 & 1.29 & & 1 \\
\hline ER105-GS & 0.783 & $(0.563-1.003)$ & 2 & 300 & $(270-330)$ & 2 & 4.87 & $(3.77-5.97)$ & 2 \\
\hline ER106-GS & 1.065 & & 1 & 320 & & 1 & 5.90 & & 1 \\
\hline ER107-GS & 0.417 & & 1 & 34.0 & & 1 & 2.98 & & 1 \\
\hline ER108-GS & 1.481 & & 1 & 690 & & 1 & 5.88 & & 1 \\
\hline ER09 & 0.680 & 0.14 & 4 & 122 & 16.9 & 4 & 6.82 & 0.42 & 4 \\
\hline ER10 & 0.561 & 0.06 & 4 & 76.5 & 15.5 & 4 & 5.53 & 0.65 & 4 \\
\hline ER15M & 0.455 & 0.04 & 6 & 74.7 & 8.3 & 6 & 5.45 & 0.40 & 6 \\
\hline ER30 & 0.378 & & 1 & 71.5 & & 1 & 3.84 & & 1 \\
\hline ER31 & 0.439 & & 1 & 76.1 & & 1 & 4.00 & & 1 \\
\hline ER32 & 0.947 & & 1 & 192 & & 1 & 7.15 & & 1 \\
\hline ER36 & 0.568 & & 1 & 229 & & 1 & 7.88 & & 1 \\
\hline ER37 & 0.655 & & 1 & 136 & & 1 & 7.22 & & 1 \\
\hline ER38 & 0.792 & & 1 & 72.9 & & 1 & 7.22 & & 1 \\
\hline ER43 & 1.123 & 0.61 & 6 & 260 & 35.2 & 6 & 9.11 & 0.52 & 6 \\
\hline ER60 & 1.195 & & 1 & 580 & & 1 & 5.91 & & 1 \\
\hline ER61 & 1.865 & 0.47 & 5 & 728 & 69.9 & 5 & 6.77 & 0.66 & 5 \\
\hline ER63 & 0.765 & 0.09 & 4 & 128 & 42.6 & 4 & 6.16 & 0.21 & 4 \\
\hline ER64 & 0.721 & $(0.648-0.794)$ & 2 & 189 & $(151-227)$ & 2 & 5.76 & $(3.18-8.33)$ & 2 \\
\hline ER73 & 0.501 & & 1 & 206 & & 1 & 7.98 & & 1 \\
\hline ER78M & 0.737 & 0.23 & 5 & 175 & 16.9 & 5 & 10.5 & 7.16 & 5 \\
\hline ER91M & 1.258 & 0.40 & 7 & 624 & 41.1 & 7 & 6.55 & 0.71 & 7 \\
\hline ER93B & 0.340 & 0.03 & 4 & 39.0 & 2.9 & 4 & 4.06 & 0.56 & 4 \\
\hline ER95B & 1.189 & 1.76 & 4 & 456 & 669 & 4 & 6.24 & 4.29 & 4 \\
\hline ERFE & 0.823 & $(0.807-0.838)$ & 2 & 372 & $(367-377)$ & 2 & 6.44 & $(5.90-6.98)$ & 2 \\
\hline ERFO & 0.689 & $(0.476-0.902)$ & 2 & 147 & $(146-147)$ & 2 & 6.34 & $(6.30-6.37)$ & 2 \\
\hline HU06 & 0.898 & 0.43 & 3 & 74.3 & 20.5 & 3 & 7.47 & 1.90 & 3 \\
\hline HU32 & 0.344 & 0.18 & 4 & 70.3 & 31.5 & 4 & 9.19 & 0.80 & 4 \\
\hline HU38 & 0.443 & 0.44 & 4 & 64.8 & 17.6 & 4 & 7.59 & 0.65 & 4 \\
\hline
\end{tabular}


Table S7, continued.

\begin{tabular}{|c|c|c|c|c|c|c|c|c|c|}
\hline Site Name & SMeHg & $\begin{array}{c}\text { Standard } \\
\text { Deviation } \\
\text { (range) }\end{array}$ & $\mathbf{n}$ & SHgT & $\begin{array}{c}\text { Standard } \\
\text { Deviation } \\
\text { (range) }\end{array}$ & $\mathbf{n}$ & LOI & $\begin{array}{c}\text { Standard } \\
\text { Deviation } \\
\text { (range) }\end{array}$ & n \\
\hline HU45M & 0.092 & & 1 & 20.2 & $(13.0-27.4)$ & 2 & 3.31 & $(1.49-5.12)$ & 2 \\
\hline HU48 & 0.223 & 0.10 & 4 & 49.3 & 18.7 & 4 & 7.5 & 0.40 & 4 \\
\hline HU54M & 0.231 & 0.29 & 4 & 23.7 & 7.6 & 4 & 3.2 & 1.15 & 4 \\
\hline HU61 & 0.099 & 0.09 & 4 & 37.5 & 26.3 & 4 & 4.9 & 2.96 & 4 \\
\hline HU93 & 1.455 & 0.38 & 4 & 106 & 12.0 & 4 & 8.2 & 0.58 & 4 \\
\hline HU95B & 0.252 & 0.14 & 4 & 43.3 & 28.8 & 4 & 6.8 & 3.11 & 4 \\
\hline HU96B & 0.278 & 0.26 & 4 & 28.9 & 20.5 & 4 & 4.2 & 2.45 & 4 \\
\hline HU97B & 0.085 & 0.003 & 3 & 9.82 & 1.8 & 4 & 3.7 & 4.76 & 4 \\
\hline HUFE & 0.080 & & 1 & 21.2 & & 1 & 3.1 & & 1 \\
\hline HUFO & 0.054 & $(0.050-0.057)$ & 2 & 5.96 & $(4.34-7.59)$ & 2 & 0.8 & $(0.46-1.07)$ & 2 \\
\hline MI101-GS & & & & 23.3 & & 1 & 2.9 & & 1 \\
\hline MI102-GS & 0.166 & & 1 & 17.7 & & 1 & 4.3 & & 1 \\
\hline MI103-GS & 0.209 & & 1 & 9.55 & & 1 & 2.7 & & 1 \\
\hline MI105-GS & 0.058 & & 1 & 3.18 & & 1 & 1.0 & & 1 \\
\hline MI106-GS & 0.006 & & 1 & 2.12 & & 1 & 2.2 & & 1 \\
\hline MI107-GS & 0.058 & & 1 & 11.4 & & 1 & 1.1 & & 1 \\
\hline MI108-GS & & & & 126 & & 1 & 9.4 & & 1 \\
\hline MI112-GS & 0.657 & & 1 & & & & 12.8 & & 1 \\
\hline MI113-GS & 0.642 & & 1 & 86.2 & & 1 & 7.0 & & 1 \\
\hline MI114-GS & 0.147 & & 1 & 42.9 & & 1 & 8.4 & & 1 \\
\hline MI115-GS & 2.072 & & 1 & 297 & & 1 & 18.7 & & 1 \\
\hline MI116-GS & 5.403 & & 1 & 942 & & 1 & 17.5 & & 1 \\
\hline MI117-GS & 0.020 & & 1 & 4.01 & & 1 & 1.4 & & 1 \\
\hline MI118-GS & 0.037 & & 1 & 2.22 & & 1 & 3.2 & & 1 \\
\hline MI119-GS & 0.052 & & 1 & 1.58 & & 1 & 1.4 & & 1 \\
\hline MI120-GS & 0.049 & & 1 & 2.72 & & 1 & 2.8 & & 1 \\
\hline MI11 & 0.318 & 0.31 & 3 & 65.4 & 49.2 & 3 & 6.9 & 2.35 & 3 \\
\hline MI18M & 0.755 & 0.43 & 6 & 136 & 11.6 & 6 & 9.8 & 0.89 & 6 \\
\hline MI27M & 0.036 & 0.03 & 4 & 9.05 & 1.5 & 4 & 1.8 & 0.39 & 4 \\
\hline MI30B & 0.109 & 0.10 & 4 & 3.77 & 3.4 & 4 & 0.6 & 0.45 & 4 \\
\hline MI31B & 0.075 & 0.08 & 4 & 8.27 & 4.4 & 4 & 1.6 & 0.61 & 4 \\
\hline MI34 & 0.239 & & 1 & 78.2 & & 1 & 5.8 & & 1 \\
\hline MI40 & 0.240 & 0.29 & 4 & 31.5 & 23.9 & 4 & 4.2 & 0.92 & 4 \\
\hline MI41M & 0.928 & 0.64 & 4 & 126 & 58.3 & 4 & 9.7 & 1.02 & 4 \\
\hline MI42B & 0.121 & 0.08 & 4 & 6.99 & 1.6 & 4 & 1.5 & 0.65 & 4 \\
\hline MI46B & 0.538 & 0.17 & 4 & 67.7 & 12.6 & 4 & 4.9 & 2.06 & 4 \\
\hline MI47 & 1.803 & 0.45 & 6 & 181 & 25.5 & 6 & 10.0 & 1.01 & 6 \\
\hline MI49B & 0.115 & 0.07 & 6 & 35.5 & 15.4 & 6 & 4.0 & 2.20 & 6 \\
\hline
\end{tabular}


271 Table S7, continued.

\begin{tabular}{|c|c|c|c|c|c|c|c|c|c|}
\hline Site Name & SMeHg & $\begin{array}{c}\text { Standard } \\
\text { Deviation } \\
\text { (range) }\end{array}$ & $\mathbf{n}$ & SHgT & $\begin{array}{c}\text { Standard } \\
\text { Deviation } \\
\text { (range) }\end{array}$ & $\mathbf{n}$ & LOI & $\begin{array}{c}\text { Standard } \\
\text { Deviation } \\
\text { (range) }\end{array}$ & $\mathbf{n}$ \\
\hline MI50B & 1.904 & 0.93 & 5 & 429 & 165 & 6 & 17.9 & 2.04 & 6 \\
\hline MI51B & 0.119 & 0.08 & 4 & 35.1 & 23.1 & 4 & 3.3 & 1.61 & 4 \\
\hline MI52B & 0.079 & 0.04 & 4 & 10.1 & 8.0 & 4 & 1.5 & 0.77 & 4 \\
\hline MI53B & 0.157 & 0.12 & 4 & 27.8 & 17.6 & 4 & 3.5 & 1.59 & 4 \\
\hline MIFE & 1.086 & & 1 & 98.5 & & 1 & 7.0 & & 1 \\
\hline MIFO & 0.049 & $\begin{array}{c}(0.046- \\
0.051)\end{array}$ & 2 & 15.5 & $(12.8-18.1)$ & 2 & 5.4 & $(3.87-7.01)$ & 2 \\
\hline ON25 & 2.382 & 1.71 & 4 & 850 & 280 & 4 & 9.3 & 2.80 & 4 \\
\hline ON33M & 1.205 & $(1.01-1.40)$ & 2 & 200 & $(150-251)$ & 2 & 7.7 & $(7.61-7.70)$ & 2 \\
\hline ON41 & 3.861 & 0.99 & 4 & 1160 & 379 & 4 & 9.9 & 1.97 & 4 \\
\hline ON55M & 4.631 & 2.46 & 5 & 1397 & 816 & 5 & 8.7 & 2.99 & 5 \\
\hline ON60 & 4.964 & 2.20 & 4 & 1618 & 388 & 4 & 11.0 & 0.69 & 4 \\
\hline ON63 & 2.847 & 0.69 & 4 & 1174 & 257 & 4 & 11.5 & 1.10 & 4 \\
\hline ON64B & 0.863 & 0.76 & 3 & 299 & 270 & 3 & 2.7 & 1.29 & 3 \\
\hline ON65B & 0.082 & 0.07 & 3 & 33.4 & 11.6 & 3 & 0.7 & 0.15 & 3 \\
\hline ON67B & 0.701 & $\begin{array}{c}(0.668- \\
0.734)\end{array}$ & 2 & 163 & $(160-165)$ & 2 & 8.1 & $(7.98-8.17)$ & 2 \\
\hline ON68B & 0.764 & 0.60 & 4 & 290 & 270 & 4 & 6.5 & 0.22 & 4 \\
\hline ON69B & 0.520 & & 1 & 274 & & 1 & 1.3 & & 1 \\
\hline ONFO & 0.422 & & 1 & 154 & & 1 & 6.8 & & 1 \\
\hline SU01M & 0.012 & 0.01 & 3 & 13.6 & 3.4 & 3 & 1.2 & 0.29 & 3 \\
\hline SU02 & 0.009 & & 1 & 55.2 & & 1 & & & \\
\hline SU09 & 0.328 & & 1 & 87.3 & & 1 & 8.2 & & 1 \\
\hline SU10 & 0.415 & 0.31 & 5 & 113 & 12.7 & 5 & 6.7 & 0.49 & 3 \\
\hline SU12 & & & & 54.2 & & 1 & & & \\
\hline SU13 & 0.294 & 0.36 & 4 & 93.7 & 39.5 & 4 & 7.7 & 1.44 & 3 \\
\hline SU15 & 1.902 & 1.75 & 4 & 225 & 55.7 & 4 & 7.8 & 0.42 & 4 \\
\hline SU16 & 0.096 & 0.06 & 4 & 82.4 & 37.3 & 4 & 7.1 & 2.27 & 4 \\
\hline SU17M & 0.157 & 0.14 & 3 & 69.2 & 24.7 & 4 & 7.3 & 0.19 & 4 \\
\hline SU18 & & & & 26.4 & & 1 & & & \\
\hline SU19 & 0.064 & 0.06 & 4 & 78.8 & 37.4 & 4 & 7.4 & 2.01 & 4 \\
\hline SU20B & 0.057 & 0.08 & 5 & 20.2 & 1.3 & 5 & 4.8 & 3.19 & 5 \\
\hline SU21B & 0.054 & 0.03 & 4 & 74.5 & 32.8 & 4 & 5.2 & 1.18 & 4 \\
\hline SU22B & 0.456 & 0.21 & 4 & 112 & 29.4 & 4 & 6.6 & 0.51 & 4 \\
\hline SU23B & 0.041 & 0.04 & 3 & 13.0 & 4.1 & 3 & 1.1 & 0.15 & 3 \\
\hline SUFO & 0.057 & $\begin{array}{c}(0.018- \\
0.096)\end{array}$ & 2 & 9.44 & $(8.53-10.4)$ & 2 & 1.4 & $(1.26-1.60)$ & 2 \\
\hline
\end{tabular}


273 Table S8. Average dissolved organic carbon (DOC), particulate-bound total mercury (PHgT) concentration

274 and partitioning coefficient $(\log \mathrm{Kd})$, particulate-bound methylmercury $(\mathrm{PMeHg})$ concentration and

275 partitioning coefficient $(\log \mathrm{Kd})$. Variability measured as standard deviation (SD) across all measurements.

276

277

\begin{tabular}{|c|c|c|c|c|c|c|c|c|c|c|c|c|}
\hline Lake & $\begin{array}{r}\text { DOC } \\
\mathrm{m} \\
\end{array}$ & $\begin{array}{l}S D \\
L^{-1}\end{array}$ & $\begin{array}{r}\text { SPM } \\
\mathrm{m} \\
\end{array}$ & $S D$ & $\begin{array}{r}\text { PHgT } \\
\text { ng } \\
\end{array}$ & $\begin{array}{c}S D \\
\mathrm{~g}^{-1} \\
\end{array}$ & $\begin{array}{r}\mathrm{PMeH} \\
\mathrm{ng} \\
\end{array}$ & & $\begin{array}{r}\log K_{d} P \\
\log (1 \\
\end{array}$ & $S D$ & $\begin{array}{r}\log K_{d} P N \\
\log (1 \\
\end{array}$ & $S D$ \\
\hline Erie - Spring & 2.14 & 0.03 & 10.5 & 6.2 & 249 & 249 & 3.2 & 2.7 & 5.8 & 0.5 & 5.4 & 0.6 \\
\hline Erie - Summer & 2.51 & 0.04 & 1.76 & 1.6 & 436 & 402 & 5.4 & 5.0 & 6.2 & 0.4 & 5.5 & 0.4 \\
\hline Huron & 1.65 & 0.08 & 0.24 & 0.09 & 154 & 39 & 5.3 & 4.7 & 5.9 & 0.0 & 5.9 & 0.4 \\
\hline Michigan & 1.86 & 0.13 & 0.33 & 0.11 & 108 & 43 & 5.4 & 4.1 & 5.8 & 0.2 & 6.0 & 0.3 \\
\hline Ontario & 2.09 & 0.07 & 0.58 & 0.20 & 84 & 58 & 5.8 & 6.8 & 5.7 & 0.3 & 5.9 & 0.5 \\
\hline Superior & 1.26 & 0.04 & 0.29 & 0.06 & 115 & 20 & 3.6 & 2.0 & 5.9 & 0.1 & 5.8 & 0.4 \\
\hline
\end{tabular}

278

279

280

281 
282 Table S9. Data availability for Lakes Michigan, Huron, Erie, Ontario and Superior (LM, LH, LE, 283 LO, and LS, respectively) provided by the USGS Mercury Research Lab and similar work

284 previously presented. ${ }^{7,11-16}$ Analytical codes are as follows: $\mathrm{U}=$ unfiltered; $\mathrm{P}=$ Particulate-bound;

$285 \mathrm{~B}=$ Biological; $\mathrm{S}=$ Sediment; $\mathrm{PW}=$ Porewater; MeHg = Methylmercury; $\mathrm{HgT}=$ Total mercury;

$286 \mathrm{HgR}=$ Reduceable mercury; DGM = Dissolved gaseous mercury; SPM = Suspended particulate

287 matter; DOC $=$ Dissolved organic matter; LOI = Loss on ignition; SUVA = Specific ultraviolet

288 absorbance.

\begin{tabular}{|c|c|c|c|c|c|}
\hline Season & Lakes & Measurements in water & Measurements in biota & Measurements in sediments & Notes \\
\hline Summer 1994 & LM & $\begin{array}{l}\text { UHgT, PHgT, PMeHg, } \\
\text { UMeHg, DGM, SPM }\end{array}$ & BHgT, BMeHg & SHgT, SMeHg & $\begin{array}{l}\text { Mason and Sullivan } 1997 \\
\text { and Sullivan and Mason } \\
1998\end{array}$ \\
\hline Fall 1994 & LM & $\begin{array}{l}\text { UHgT, PHgT, PMeHg, } \\
\text { UMeHg, DGM, SPM }\end{array}$ & $\mathrm{BHgT}$, BMeHg & SHgT, SMeHg & $\begin{array}{l}\text { Mason and Sullivan } 1997 \\
\text { and Sullivan and Mason } \\
1998\end{array}$ \\
\hline Spring 1995 & LM & $\begin{array}{l}\text { UHgT, PHgT, PMeHg, } \\
\text { UMeHg, DGM, SPM }\end{array}$ & BHgT, BMeHg & SHgT, SMeHg & $\begin{array}{l}\text { Mason and Sullivan } 1997 \\
\text { and Sullivan and Mason } \\
1998\end{array}$ \\
\hline Fall 1995 & LM & $\begin{array}{l}\text { UHgT, PHgT, PMeHg, } \\
\text { UMeHg, DGM, SPM }\end{array}$ & BHgT, BMeHg & SHgT, SMeHg & $\begin{array}{c}\text { Mason and Sullivan } 1997 \\
\text { and Sullivan and Mason } \\
1998 \\
\end{array}$ \\
\hline Spring 2000 & LS & $\begin{array}{c}\text { UHgT, PHgT, PMeHg, } \\
\text { DOC, SUVA, SPM }\end{array}$ & & & Rolfhus et al. 2003 \\
\hline Summer 2000 & LS & $\begin{array}{c}\text { UHgT, PHgT, PMeHg, } \\
\text { UMeHg, HgRR }{ }^{1} \text {, DGM, DOC, } \\
\text { SUVA, SPM }\end{array}$ & & PWHgT, SHgT, SMeHg & Rolfhus et al. 2003 \\
\hline Spring 2005 & LM & UHgT, UMeHg, DGM & & & $\begin{array}{l}\text { Jeremiason et al. } \\
2009\end{array}$ \\
\hline Summer 2005 & LM & UHgT, UMeHg, DGM & & & $\begin{array}{c}\text { Jeremiason et al. } \\
2009\end{array}$ \\
\hline Spring 2006 & LM & UHgT, UMeHg, DGM & & & $\begin{array}{l}\text { Jeremiason et al. } \\
2009\end{array}$ \\
\hline Summer 2006 & LM & UHgT, UMeHg, DGM & & & $\begin{array}{c}\text { Jeremiason et al. } \\
2009\end{array}$ \\
\hline Summer 2010 & $\mathrm{LM}, \mathrm{LH}$ & $\begin{array}{c}\mathrm{FHgT}, \mathrm{FMeHg}^{2}, \mathrm{PHgT} \text {, } \\
\mathrm{FMeHg}\end{array}$ & $\mathrm{BHgT}^{5}, \mathrm{BMeHg}^{5}$ & SHgT, SMeHg, LOI, Dry weight & \\
\hline Summer 2010 & LS, LE, LO & UHgT, UMeHg ${ }^{2}$ & $\mathrm{BHgT}^{5}, \mathrm{BMeHg}^{5}$ & SHgT, SMeHg, LOI, Dry weight & \\
\hline Spring 2011 & LS, LH, LM, LH, LE, LO & UHgT, UMeHg ${ }^{2}$, DOC & $\mathrm{BHgT}^{3}, \mathrm{BMeHg}^{3}$ & SHgT, SMeHg, LOI, Dry weight & \\
\hline Spring 2011 & Western LE & $\begin{array}{c}\mathrm{FHgT}, \mathrm{FMeHg}^{2}, \mathrm{PHgT}, \\
\mathrm{PMeHg}^{2}, \mathrm{DOC}\end{array}$ & $\mathrm{BHgT}^{3}, \mathrm{BMeHg}^{3}$ & SHgT, SMeHg, LOI, Dry weight & \\
\hline Summer 2011 & LS, LH, LM, LH, LE, LO & UHgT, UMeHg ${ }^{2}$, DOC & $\mathrm{BHgT}^{3,5}, \mathrm{BMeHg}^{3,5}$ & SHgT, SMeHg, LOI, Dry weight & \\
\hline Summer 2011 & Western LE & $\begin{array}{c}\mathrm{FHgT}, \mathrm{FMeHg}^{2}, \mathrm{PHgT}, \\
\text { PMeHg, DOC }\end{array}$ & $\mathrm{BHgT}^{3}, \mathrm{BMeHg}^{3}$ & SHgT, SMeHg, LOI, Dry weight & \\
\hline Spring 2012 & LS, LH, LM, LH, LE, LO & UHgT, UMeHg ${ }^{2}, \mathrm{DOC}$ & $\mathrm{BHgT}^{3}, \mathrm{BMeHg}^{3}$ & & \\
\hline Spring 2012 & Western LE & $\begin{array}{c}\text { FHgT, FMeHg }{ }^{2} \text {, PHgT, } \\
\text { PMeHg, DOC }\end{array}$ & $\mathrm{BHgT}^{3}, \mathrm{BMeHg}^{3}$ & & \\
\hline Summer 2012 & LS, LH, LM, LH, LE, LO & UHgT, UMeHg ${ }^{2}, \mathrm{DOC}$ & $\mathrm{BHgT}^{3,5}, \mathrm{BMeHg}^{3,5}$ & SHgT, SMeHg, LOI, Dry weight & \\
\hline Summer 2012 & Western LE & $\begin{array}{c}\text { FHgT, } \mathrm{FMeHg}^{2}, \mathrm{PHgT}, \\
\mathrm{PMeHg}, \mathrm{DOC}\end{array}$ & $\mathrm{BHgT}^{3}, \mathrm{BMeHg}^{3}$ & SHgT, SMeHg, LOI, Dry weight & \\
\hline Summer 2013 & LS, LH, LM, LH, LE, LO & UHgT, UMeHg${ }^{2}, \mathrm{DOC}$ & $\mathrm{BHgT}^{3,5}, \mathrm{BMeHg}^{3,5}$ & SHgT, SMeHg, LOI, Dry weight & $\begin{array}{l}\text { Partly in Lepak et al. ES\&T, } \\
2015 \text { and Lepak et al. ES\&T } \\
\text { Letters, 2015 }\end{array}$ \\
\hline Summer 2013 & Western LE & $\begin{array}{l}\text { FHgT, } \mathrm{FMeHg}^{2}, \mathrm{PHgT} \text {, } \\
\text { PMeHg, DOC }\end{array}$ & $\mathrm{BHgT}^{3}, \mathrm{BMeHg}^{3}$ & SHgT, SMeHg, LOI, Dry weight & $\begin{array}{c}\text { Partly in Lepak et al. ES\&T, } \\
2015 \text { and Lepak et al. ES\&T } \\
\text { Letters, } 2015 \\
\end{array}$ \\
\hline Fall 2013 & LM & $\begin{array}{l}\text { FHgT, } \mathrm{FMeHg}^{2}, \mathrm{PHgT}, \\
\text { PMeHg, DGM*, DOC }\end{array}$ & $\mathrm{BHgT}^{4,5}, \mathrm{BMeHg}^{4,5}$ & SHgT, SMeHg, LOI, Dry weight & $\begin{array}{l}\text { Partly in Lepak et al. } \\
\text { ES\&T Water, } 2020\end{array}$ \\
\hline Spring 2014 & LS, LH, LM, LH, LE, LO & $\begin{array}{l}\text { FHgT, } \mathrm{FMeHg}^{2} \text {, FMeHg, } \\
\text { PHgT, PMeHg, DOC }\end{array}$ & $\begin{array}{c}\mathrm{BHgT}^{4}, \mathrm{BMeHg}^{4}, \mathrm{C} \text { and } \mathrm{N} \\
\text { isotopes } \\
\end{array}$ & & $\begin{array}{c}\text { Partly in Lepak et al. ES\&T, } \\
2015 \text { and Lepar et al. ES\&T } \\
\text { Letters, 2015 }\end{array}$ \\
\hline Summer 2014 & LS, LH, LM, LH, LE, LO & $\begin{array}{l}\text { FHgT, FMeHg }{ }^{2}, F M e H g, \\
\text { PHgT, PMeHg, DOC }\end{array}$ & $\begin{array}{c}\mathrm{BHgT}^{4}, \mathrm{BMeHg}^{4}, \mathrm{C} \text { and } \mathrm{N} \\
\text { isotopes }\end{array}$ & & $\begin{array}{c}\text { Partly in Lepak et al. ES\&T, } \\
2015 \text { and Lepak et al. ES\&T } \\
\text { Letters, } 2015 \\
\end{array}$ \\
\hline Fall 2014 & LE & $\begin{array}{l}\text { FHgT, FMeHg }{ }^{2} \text {, FMeHg, } \\
\text { PHgT, PMeHg, DOC, SPM }\end{array}$ & $\begin{array}{c}\mathrm{BHgT}^{4}, \mathrm{BMeHg}^{4}, \mathrm{C} \text { and N } \\
\text { isotopes }\end{array}$ & SHgT, SMeHg, LOI, Dry weight & \\
\hline Spring 2018 & LS, LH, LM, LH, LE, LO & FMeHg, DOC, SPM & $\begin{array}{l}\mathrm{BHgT}^{4}, \mathrm{BMeH}^{4}, \mathrm{C} \text { and } \mathrm{N} \\
\text { isotopes }{ }^{4} \text {, biomass est. }\end{array}$ & & \\
\hline Fall 2018 & LS, LH, LM, LH, LE, LO & FMeHg, DOC, SPM & $\begin{array}{l}\mathrm{BHgT}^{4}, \mathrm{BMeHg}^{4}, \mathrm{C} \text { and } \mathrm{N} \\
\text { isotopes }{ }^{4}, \text { biomass est }{ }^{4} \text {. }\end{array}$ & & \\
\hline $\begin{array}{l}\text { RR in Rolfhus et al. } 2003 \\
\text { eHg analyses (UMeHg ar } \\
\text { se methods were not us } \\
\text { halysis of seston, not size } \\
\text { halysis of seston, conduc }\end{array}$ & $\begin{array}{l}\text { ures purgable gaseous mercury } \\
\text { leHg) were performed using co } \\
\text { r data interpretation. } \\
\text { arated, or separated at 63-118н } \\
\text { in seston tissue size separated a } \\
\text { issue }\end{array}$ & $\begin{array}{l}\text { ry without the aid of intitial chemical ox } \\
\text { onventional small volume distillation b } \\
\text { im and }>118 \mu \mathrm{m} \text { size fractions; not used for d } \\
\text { at } 63-118 \mu \mathrm{m}, 118-243 \mu \mathrm{m}, 243-500 \mu \mathrm{m} \text {, an }\end{array}$ & $\begin{array}{l}\text { tope dilution. Due to insufic } \\
\text { terpretation. } \\
\text { ofum. }\end{array}$ & lyte detection, LS, LH, LM, LE (main la & LO water values using \\
\hline
\end{tabular}


Table S10. Interlaboratory comparison for measurement of carbon and nitrogen content and isotope ratios of in-house standard and field collected triplicate precision. Variability reported as the average of one standard deviation (SD) or two standard error (SE).

\begin{tabular}{|c|c|c|c|c|c|}
\hline \multicolumn{6}{|c|}{ Mendota 243 micron seston lab standard } \\
\hline \multirow{2}{*}{\multicolumn{6}{|c|}{$\begin{array}{l}\text { U.S. Environmental Protection Agency Great Lakes Toxicology and Ecology } \\
\text { Division, Minnesota }\end{array}$}} \\
\hline & & & & & \\
\hline & $\delta^{15} \mathrm{~N}$ & $\delta^{13} \mathrm{C}$ & Percent as C & Percent as N & $\mathrm{C}: \mathrm{N}_{\text {mass ratio }}$ \\
\hline Ave & 16.7 & -30.6 & 55.8 & 10.8 & 6.2 \\
\hline SD & 0.2 & 0.1 & 3.00 & 1.4 & 0.5 \\
\hline $2 S E(n=44)$ & 0.1 & 0.0 & 0.9 & 0.4 & 0.1 \\
\hline \multicolumn{6}{|c|}{$\begin{array}{l}\text { Great Lakes Institute for Environmental Research, at the University of } \\
\text { Windsor, Ontario }\end{array}$} \\
\hline Ave & 16.7 & -31.7 & 51.7 & 9.8 & 5.3 \\
\hline SD & 0.1 & 0.1 & 2.2 & 0.4 & 0.1 \\
\hline 2SE $(n=44)$ & 0.1 & 0.1 & 1.0 & 0.2 & 0.0 \\
\hline \multicolumn{6}{|c|}{ Field triplicate precision } \\
\hline Ave $(n=15)$ & 0.2 & 0.1 & 1.4 & 0.3 & 0.1 \\
\hline
\end{tabular}

294 Citations

295 1. Epa, U., Method 1630, Methyl mercury in water by distillation, aqueous ethylation, purge 296 and trap, and CVAFS. US Environmental Protection Agency, Washington, DC 1998.

$2972 . \quad$ Agency., U. E. P., Mercury in solids and solutions by thermal decomposition, 298 amalgamation, and atomic absorption spectrophotometry. Method 7473. In US Environmental 299 Protection Agency Washington, DC 1998.

$3003 . \quad$ Ogorek, J. M., Data release for: Enhanced sensitivity of methylmercury bioaccumulation 301 into seston of the Laurentian Great Lakes. U.S. Geological Survey Data Release, U.S.

302 Geoloogical Survey 2021.

303 4. Weishaar, J. L.; Aiken, G. R.; Bergamaschi, B. A.; Fram, M. S.; Fujii, R.; Mopper, K., 304 Evaluation of specific ultraviolet absorbance as an indicator of the chemical composition and 305 reactivity of dissolved organic carbon. Environ. Sci. Technol. 2003, 37, (20), 4702-4708.

3065 5. Epa, U., Method 1631, Revision E: Mercury in water by oxidation, purge and trap, and 307 cold vapor atomic fluorescence spectrometry. US Environmental Protection Agency Washington, 308 DC 2002.

309 6. Hammerschmidt, C. R.; Fitzgerald, W. F., Bioaccumulation and trophic transfer of 310 methylmercury in Long Island Sound. Arch. Environ. Con. Tox. 2006, 51, (3), 416-424.

311 7. Lepak, R. F.; Krabbenhoft, D. P.; Ogorek, J. M.; Tate, M. T.; Bootsma, H. A.; Hurley, J. 312 P., Influence of cladophora-quagga mussel assemblages on nearshore methylmercury production in Lake Michigan. Environ. Sci. Technol. 2015, 49, (13), 7606-7613.

8. Mansfield, C. R.; Black, F. J., Quantification of monomethylmercury in natural waters by direct ethylation: Interference characterization and method optimization. Limnol. OceanogrMeth. 2015, 13, (2), 81-91.

9. Federation, W. E.; Association, A., Standard methods for the examination of water and wastewater. American Public Health Association (APHA): Washington, DC, USA 2005.

10. Sierszen, M. E.; Hrabik, T. R.; Stockwell, J. D.; Cotter, A. M.; Hoffman, J. C.; Yule, D. L., Depth gradients in food-web processes linking habitats in large lakes: Lake Superior as an exemplar ecosystem. Freshw. Biol. 2014, 59, (10), 2122-2136. 
322 11. Mason, R. P.; Sullivan, K. A., Mercury in lake Michigan. Environ. Sci. Technol. 1997, 323 31, (3), 942-947.

324 12. Sullivan, K. A.; Mason, R. P., The concentration and distribution of mercury in Lake 325 Michigan. Sci. Total Environ. 1998, 213, (1-3), 213-228.

326 13. Jeremiason, J. D.; Kanne, L. A.; Lacoe, T. A.; Hulting, M.; Simcik, M. F., A comparison 327 of mercury cycling in Lakes Michigan and Superior. J. Great Lakes Res. 2009, 35, (3), 329-336.

$328 \quad$ 14. Lepak, R. F.; Tate, M. T.; Ogorek, J. M.; DeWild, J. F.; Peterson, B. D.; Hurley, J. P.; 329 Krabbenhoft, D. P., Aqueous Elemental Mercury Production versus Mercury Inventories in the 330 Lake Michigan Airshed: Deciphering the Spatial and Diel Controls of Mercury Gradients in Air 331 and Water. ACS Environ. Sci. Technol. Water 2020.

$332 \quad 15 . \quad$ Rolfhus, K.; Sakamoto, H.; Cleckner, L.; Stoor, R.; Babiarz, C.; Back, R.; Manolopoulos, 333 H.; Hurley, J., Distribution and fluxes of total and methylmercury in Lake Superior. Environ. Sci. 334 Technol. 2003, 37, (5), 865-872.

335 16. Lepak, R. F.; Yin, R.; Krabbenhoft, D. P.; Ogorek, J. M.; DeWild, J. F.; Holsen, T. M.; 336 Hurley, J. P., Use of stable isotope signatures to determine mercury sources in the Great Lakes. 337 Environ. Sci. Technol. Let. 2015, 2, (12), 335-341. 